\title{
ON IDEALS IN THE ENVELOPING ALGEBRA OF A LOCALLY SIMPLE LIE ALGEBRA
}

\author{
IVAN PENKOV AND ALEXEY PETUKHOV
}

\begin{abstract}
We study (two-sided) ideals $I$ in the enveloping algebra $\mathrm{U}\left(\mathfrak{g}_{\infty}\right)$ of an infinite-dimensional Lie algebra $\mathfrak{g}_{\infty}$ obtained as the union (equivalently, direct limit) of an arbitrary chain of embeddings of simple finite-dimensional Lie algebras

$$
\mathfrak{g}_{1} \rightarrow \mathfrak{g}_{2} \rightarrow \ldots \rightarrow \mathfrak{g}_{n} \rightarrow \ldots
$$

with $\lim _{n \rightarrow \infty} \operatorname{dim} \mathfrak{g}_{n}=\infty$. Our main result is an explicit description of the zerosets of the corresponding graded ideals gr $I$. We use this description and results of A. Zhilinskii to prove Baranov's conjecture that, if $\mathfrak{g}_{\infty}$ is not diagonal in the sense of A. Baranov and A. Zhilinskii, then $\mathrm{U}\left(\mathfrak{g}_{\infty}\right)$ admits a single non-zero proper ideal: the augmentation ideal.

Our study is based on a complete description of the radical Poisson ideals in $\mathbf{S}\left(\mathfrak{g}_{\infty}\right)$ and their zero-sets. We then discuss in detail integrable ideals of $\mathrm{U}\left(\mathfrak{g}_{\infty}\right)$, i.e. ideals $I \subset \mathrm{U}\left(\mathfrak{g}_{\infty}\right)$ for which $I \cap \mathrm{U}\left(\mathfrak{g}_{n}\right)$ is an intersection of ideals of finite-codimension in $\mathrm{U}\left(\mathfrak{g}_{n}\right)$ for any $n \geq 1$. We present a classification of prime integrable ideals based on work of A. Zhilinskii. For $\mathfrak{g}_{\infty} \cong \mathfrak{s l}_{\infty}, \mathfrak{s o}_{\infty}$, all zero-sets of radical Poisson ideals of $\mathbf{S}\left(\mathfrak{g}_{\infty}\right)$ arise from prime integrable ideals of $\mathrm{U}\left(\mathfrak{g}_{\infty}\right)$. For $\mathfrak{g}_{\infty} \cong \mathfrak{s p}_{\infty}$ only "half" of the zero-sets of Poisson ideals $\mathbf{S} \cdot\left(\mathfrak{g}_{\infty}\right)$ arise from integrable ideals of $\mathrm{U}\left(\mathfrak{g}_{\infty}\right)$.

Key words: associated variety, coherent local system, integrable ideal, locally finite Lie algebra, moment map, Poisson ideal.

AMS subject classification. Primary: 14L30, 17B35, 17B63, $17 \mathrm{~B} 65$.
\end{abstract}

\section{INTRODUCTION}

We work over an algebraically closed field of characteristic zero. A locally finite Lie algebra is by definition a Lie algebra isomorphic to the limit of some system of finite-dimensional Lie algebras $\mathrm{BS}$. In what follows we restrict ourselves to locally finite Lie algebras $\mathfrak{g}_{\infty}$ defined as direct limits of respective sequences of embeddings of simple Lie algebras

$$
\mathfrak{g}_{1} \rightarrow \mathfrak{g}_{2} \rightarrow \ldots \rightarrow \mathfrak{g}_{n} \rightarrow \ldots
$$

such that $\lim _{n \rightarrow \infty} \operatorname{dim} \mathfrak{g}_{n}=\infty$; for brevity we refer just to these Lie algebras as locally simple Lie algebras.

Locally simple Lie algebras are natural infinite-dimensional analogs of finitedimensional simple Lie algebras, and their representation theory is interesting and challenging.

In this paper we study ideals in the universal enveloping algebra $U\left(\mathfrak{g}_{\infty}\right)$ of a locally simple Lie algebra $\mathfrak{g}_{\infty}$, in particular annihilators of simple $\mathfrak{g}_{\infty}$-modules, i.e. primitive ideals. The structure of ideals in $U\left(\mathfrak{g}_{\infty}\right)$ differs significantly from the structure of ideals in the enveloping algebra of a simple finite-dimensional Lie 
algebra. This is not surprising once one observes that the center of $U\left(\mathfrak{g}_{\infty}\right)$ consists of constants only.

The theory of ideals in $\mathrm{U}\left(\mathfrak{g}_{\infty}\right)$ has been initiated by A. Zhilinskii in [Zh1, [Zh2]. His method is to study "coherent local systems" of finite-dimensional modules of the sequence of Lie algebras $\mathfrak{g}_{n}$ and to construct ideals in $\mathrm{U}\left(\mathfrak{g}_{\infty}\right)$ whose intersections with $\mathrm{U}\left(\mathfrak{g}_{n}\right)$ are the joint annihilators of the modules at the $n$-th level of the respective local systems. We call the ideals of $\mathrm{U}\left(\mathfrak{g}_{\infty}\right)$ arising in this way integrable ideals.

A central idea of the present paper is to gain information about ideals in $\mathrm{U}\left(\mathfrak{g}_{\infty}\right)$ by studying their associated "varieties". At first this leads to the study of radical Poisson ideals in the symmetric algebra $\mathbf{S}\left(\mathfrak{g}_{\infty}\right)$. Our first notable result is that $\mathbf{S}\left(\mathfrak{g}_{\infty}\right)$ admits a non-zero Poisson ideal $J$ of locally infinite codimension (i.e. such that $J \cap \mathbf{S}\left(\mathfrak{g}_{n}\right)$ is of infinite codimension in $\mathbf{S}\left(\mathfrak{g}_{n}\right)$ for almost all $n$ ) if and only if $\mathfrak{g}_{\infty}$ is isomorphic to one of the three locally simple Lie algebras $\mathfrak{s l}_{\infty}, \mathfrak{s o}_{\infty}, \mathfrak{s p}_{\infty}$.

This result, together with results of A. Zhilnskii [Zh2, yields the following corollary: if $\mathfrak{g}$ is not isomorphic to $\mathfrak{s l}_{\infty}, \mathfrak{s o}_{\infty}, \mathfrak{s p} \mathfrak{p}_{\infty}$, and $\mathrm{U}\left(\mathfrak{g}_{\infty}\right)$ has a non-zero proper ideal $I$ which does not coincide with the augmentation ideal, then $I$ is of locally finite codimension (i.e. $I \cap \mathrm{U}\left(\mathfrak{g}_{n}\right)$ is of finite codimension in $\mathrm{U}\left(\mathfrak{g}_{n}\right)$ for all $n$ ) and $\mathfrak{g}_{\infty}$ is diagonal. Diagonal locally simple Lie algebras form a natural class of locally simple Lie algebras which has been introduced by A. Baranov and A. Zhilinskii; in fact, these authors have given an intricate and elegant classification of diagonal locally simple Lie algebras BZh. Moreover, the above corollary had been conjectured by A. Baranov.

As a second notable result on Poisson ideals we compute the set of zeros $\operatorname{Var}(J)$ (i.e. the associated "variety") of any Poisson ideal $J \subset \mathbf{S}\left(\mathfrak{g}_{\infty}\right)$ for $\mathfrak{g}_{\infty}=\mathfrak{s l}_{\infty}, \mathfrak{s o}_{\infty}, \mathfrak{s p}_{\infty}$.

We then pass to the study of ideals in $\mathrm{U}\left(\mathfrak{g}_{\infty}\right)$ for $\mathfrak{g}_{\infty}=\mathfrak{s l}_{\infty}, \mathfrak{s o}_{\infty}, \mathfrak{s p}_{\infty}$. In Section 7 we review Zhilnskii's results and, most importantly, his classification of irreducible coherent local systems. Zhilinskii's results yield an explicit description of all prime integrable ideals in $\mathrm{U}\left(\mathfrak{g}_{\infty}\right)$, Theorem 7.8 .

We finally describe the associated "varieties" of integrable ideals. In particular, we show that if $\mathfrak{g}_{\infty}=\mathfrak{s l}_{\infty}, \mathfrak{s 0} \boldsymbol{0}_{\infty}$ and $J$ is a radical Poisson ideal of $\mathbf{S}\left(\mathfrak{g}_{\infty}\right)$, there exists a prime integrable ideal $I$ of $\mathrm{U}\left(\mathfrak{g}_{\infty}\right)$ such that $\operatorname{Var}(I)=\operatorname{Var}(J)$. For $\mathfrak{g}_{\infty}=\mathfrak{s p}_{\infty}$ the situation is different: given an ideal $J$, there exists an ideal $I$ of $\mathrm{U}\left(\mathfrak{g}_{\infty}\right)$ such that $\operatorname{Var}(I)=\operatorname{Var}(J)$, but $I$ is not necessarily integrable.

We thank A. Baranov for communicating to us his conjecture and also the results of A. Zhilinskii. Ivan Penkov acknowledges support from the DFG via SPP 3188 "Darstellungstheorie".

\section{Preliminaries}

We fix an algebraically closed field $\mathbb{F}$ of characteristic zero. All vector spaces (including Lie algebras) are assumed to be defined over $\mathbb{F}$. If $V$ is a vector space, $V^{*}$ stands for the dual space $\operatorname{Hom}_{\mathbb{F}}(V, \mathbb{F})$. All varieties we consider are algebraic varieties over $\mathbb{F}$ (with Zariski topology). When considering locally finite Lie algebras or their enveloping algebras we assume that any given sequence (1) consists of inclusions, so we can freely interchange $\lim _{\rightarrow} \mathfrak{g}_{n}$ with $\cup_{n} \mathfrak{g}_{n}$ and $\lim _{\rightarrow} \mathrm{U}\left(\mathfrak{g}_{n}\right)$ with $\cup_{n} \mathrm{U}\left(\mathfrak{g}_{n}\right)$.

There is no classification of general locally simple Lie algebras: a classification is only available for the so called diagonal locally simple Lie algebras, see BZh]. 
Among diagonal locally simple Lie algebras a prominent role is played by the three simple Lie algebras $\mathfrak{s l}_{\infty}, \mathfrak{s o}_{\infty}$ and $\mathfrak{s p}_{\infty}$ which can be defined as unions of the respective chains of inclusions of classical finite-dimensional Lie algebras of types $\mathfrak{s l}, \mathfrak{s o}$, or $\mathfrak{s p}$ under the obvious "left upper-corner inclusions". An important result, see [B] or $[\mathrm{BS}$, states that, up to isomorphism, these three Lie algebras are the only locally simple finitary Lie algebras, i.e. locally simple Lie algebras which admit a countable-dimensional faithful module with a basis such that the endomorphism arising from each element of the Lie algebra is given by a matrix with finitely many non-zero entries.

Let $G$ be a connected algebraic group with Lie algebra, $\mathfrak{g}$ and $I \subset \mathrm{U}(\mathfrak{g})$ be an ideal in the enveloping algebra $U(\mathfrak{g})$ of $\mathfrak{g}$ (by an ideal in a ring we mean a proper two-sided ideal). The degree filtration $\left\{\mathrm{U}(\mathfrak{g})^{\leq d}\right\}_{d \in \mathbb{Z}>0}$ on $\mathrm{U}(\mathfrak{g})$ defines the filtration $\left\{I \cap \mathrm{U}(\mathfrak{g})^{\leq d}\right\}_{d \in \mathbb{Z}_{>0}}$ on $I$. The associated graded object $\operatorname{gr} I:=\oplus_{d}\left(\left(I \cap \mathrm{U}(\mathfrak{g})^{\leq d}\right) /(I \cap\right.$ $\mathrm{U}(\mathfrak{g}) \leq d-1)$ ) is a $G$-stable ideal of gr $\mathrm{U}(\mathfrak{g})=\mathbf{S}(\mathfrak{g})$. We denote the set of zeros of $\operatorname{gr} I$ in $\mathfrak{g}^{*}$ by $\operatorname{Var}(I) \subset \mathfrak{g}^{*}$. The variety $\operatorname{Var}(I)$ is a $G$-stable subvariety of $\mathfrak{g}^{*}$ and is, by definition, the associated variety of $I$. The ideal $I$ is of finite codimension in $\mathrm{U}(\mathfrak{g})$ if and only if $\operatorname{gr} I$ is of finite codimension in $\mathbf{S}(\mathfrak{g})$. Moreover, $\operatorname{gr} I$ is of finite codimension in $\mathbf{S}^{\prime}(\mathfrak{g})$ if and only if $\operatorname{Var}(I)=0$. We note that the $\operatorname{radical} \operatorname{rad}(\operatorname{gr} I)$ of $\operatorname{gr} I$ is also $G$-stable.

It is well known that $\mathbf{S}(\mathfrak{g})$ is a Poisson algebra, i.e. $\mathbf{S}(\mathfrak{g})$ has an $\mathbb{F}$-bilinear operation

$$
\{\cdot, \cdot\}: \mathbf{S} \cdot(\mathfrak{g}) \times \mathbf{S} \cdot(\mathfrak{g}) \rightarrow \mathbf{S}(\mathfrak{g})
$$

which is a Lie bracket and is compatible with multiplication. An ideal $J \subset \mathbf{S}(\mathfrak{g})$ is Poisson whenever $\{f, J\} \subset J$ for any $f \in \mathbf{S}^{\cdot}(\mathfrak{g})$. Assume that $\mathfrak{g}$ is finite-dimensional and semisimple and let $G$ be the adjoint group of $\mathfrak{g}$. Then the radical Poisson ideals of $\mathbf{S}(\mathfrak{g})$ are in one-to-one correspondence with the $G$-stable Zariski-closed subsets of $\mathfrak{g}^{*}$. A description of such sets is presented in Bor].

Let $\mathfrak{g}_{\infty}$ be a locally simple Lie algebra. The same arguments as in the previous paragraph assign to any ideal $I \subset \mathrm{U}\left(\mathfrak{g}_{\infty}\right)$ a radical ideal $\operatorname{rad}(\operatorname{gr} I) \subset \mathbf{S}\left(\mathfrak{g}_{\infty}\right)$, which is stable under the adjoint action of $\mathfrak{g}_{\infty}$ on $\mathbf{S}\left(\mathfrak{g}_{\infty}\right)$; in what follows we call $\mathfrak{g}_{\infty}$-stable ideals of $\mathbf{S}\left(\mathfrak{g}_{\infty}\right)$ Poisson. We denote the set of zeros of $\operatorname{gr} I$ in $\mathfrak{g}_{\infty}^{*}$ by $\operatorname{Var}(I)$ and refer to $\operatorname{Var}(I)$ as the associated "variety" of $I$.

Note that $\operatorname{Var}(I)$ is a proj-variety, i.e. an inverse limit of algebraic varieties. Indeed, fix a sequence (1) with $\mathfrak{g}_{\infty}=\lim _{\rightarrow} \mathfrak{g}_{n}$ and let $\overline{\operatorname{pr}_{\mathfrak{g}_{n}} \operatorname{Var}(I)} \subset \mathfrak{g}_{n}^{*}$ be the closure of the image of $\operatorname{Var}(I)$ under the natural projection $\operatorname{pr}_{\mathfrak{g}_{n}}: \mathfrak{g}_{\infty}^{*} \rightarrow \mathfrak{g}_{n}^{*}$; by definition $\overline{\operatorname{pr}_{\mathfrak{g}_{n}} \operatorname{Var}(I)} \subset \mathfrak{g}_{n}^{*}$ is the set of zeros of $(\operatorname{gr} I) \cap \mathbf{S}\left(\mathfrak{g}_{n}\right)$ in $\mathfrak{g}_{n}^{*}$. The space $\mathfrak{g}_{\infty}^{*}$ equals the inverse limit $\lim _{\mathfrak{n}} \mathfrak{g}_{n}^{*}$, and therefore $\operatorname{Var}(I) \subset \mathfrak{g}_{\infty}^{*}$ is the inverse limit of the algebraic varieties $\overline{\operatorname{pr}_{\mathfrak{g}_{n}} \operatorname{Var}(I)}$.

An ideal $I$ is of locally finite codimension in $\mathrm{U}\left(\mathfrak{g}_{\infty}\right)$ (i.e. $I \cap \mathrm{U}\left(\mathfrak{g}_{n}\right)$ is of finite codimension in $\mathrm{U}\left(\mathfrak{g}_{n}\right)$ for all $n$ ) if and only if the ideal gr $I$ is of locally finite codimension in $\mathbf{S}\left(\mathfrak{g}_{\infty}\right)$ (i.e. $(\operatorname{gr} I) \cap \mathbf{S}^{\cdot}\left(\mathfrak{g}_{n}\right)$ is of finite codimension in $\mathbf{S}\left(\mathfrak{g}_{n}\right)$ for all $\left.n\right)$. Furthermore, $\operatorname{gr} I$ is of locally finite codimension in $\mathbf{S}\left(\mathfrak{g}_{\infty}\right)$ if and only if $\operatorname{Var}(I)=0$. We call an ideal $I$ of locally infinite codimension if $I$ is not of locally finite codimension.

The above discussion implies in particular the following.

Proposition 2.1. Assume that $\mathfrak{g}_{\infty}$ is a locally simple Lie algebra. If $U\left(\mathfrak{g}_{\infty}\right)$ admits a non-zero ideal of locally infinite codimension, then $\mathbf{S} \cdot\left(\mathfrak{g}_{\infty}\right)$ admits a non-zero Poisson ideal of locally infinite codimension. 


\section{Poisson ideals: Statement of Results}

Our first main result is the following theorem.

Theorem 3.1. If $\mathbf{S} \cdot\left(\mathfrak{g}_{\infty}\right)$ admits a non-zero Poisson ideal of locally infinite codimension, then $\mathfrak{g}_{\infty} \cong \mathfrak{s l}_{\infty}, \mathfrak{s o}_{\infty}, \mathfrak{s p}_{\infty}$.

Corollary 3.2. If $\mathrm{U}\left(\mathfrak{g}_{\infty}\right)$ admits a non-zero ideal of locally infinite codimension, then $\mathfrak{g}_{\infty} \cong \mathfrak{s l}_{\infty}, \mathfrak{s o}_{\infty}, \mathfrak{s p}{ }_{\infty}$.

Proof. The algebra $\mathbf{S}\left(\mathfrak{g}_{\infty}\right)$ admits a non-zero Poisson ideal $J$ of locally infinite codimension by Proposition 2.1 hence $\mathfrak{g}_{\infty} \cong \mathfrak{s l}_{\infty}, \mathfrak{s o}_{\infty}, \mathfrak{s p}_{\infty}$ by Theorem 3.1]

Fix now a Lie algebra $\mathfrak{g}_{\infty}=\mathfrak{s l}_{\infty}, \mathfrak{s o}_{\infty}, \mathfrak{s p}_{\infty}$ together with a chain (1) such that $\lim _{\rightarrow} \mathfrak{g}_{n}=\mathfrak{g}_{\infty}$. Without loss of generality we assume that for $n \geq 3$ all $\mathfrak{g}_{n}$ are simple and of the same type $\mathrm{A}, \mathrm{B}, \mathrm{C}$, or $\mathrm{D}$, and that $\mathrm{rkg} \mathfrak{g}_{n}=n$. By $V_{n}$ we denote a natural representation of $\mathfrak{g}_{n}$ (for $\mathfrak{g}_{n}$ of type A there are two choices of $V_{n}$ up to isomorphism). We further assume that, for $n \geq 3, V_{n+1}$ considered as a $\mathfrak{g}_{n}$-module is isomorphic to $V_{n}$ plus a trivial module.

Set

$$
\mathfrak{g}_{n}^{\leq r}:=\left\{x \in \mathfrak{g}_{n} \mid \text { there exists } \lambda \in \mathbb{F} \text { such that } \operatorname{rk}\left(X-\lambda \operatorname{Id}_{V_{n}}\right) \leq r\right\},
$$

where $X$ is considered as a linear operator on $V_{n}$. Note that $\mathfrak{g}_{n}^{\leq r}$ is a Zariski closed subset of $\mathfrak{g}_{n}$. Choosing compatible identifications $\mathfrak{g}_{n} \cong \mathfrak{g}_{n}^{*}$, we can assume that $\mathfrak{g}_{n}^{\leq r} \subset \mathfrak{g}_{n}^{*}$. Furthermore, for $\mathfrak{g}_{\infty} \cong \mathfrak{s l}_{\infty}, \mathfrak{s o}_{\infty}, \mathfrak{s p}_{\infty}$ one can check directly that the projection $\mathfrak{g}_{n+1}^{*} \rightarrow \mathfrak{g}_{n}^{*}$ maps $\mathfrak{g}_{n+1}^{\leq r}$ surjectively onto $\mathfrak{g}_{n}^{\leq r}$. This yields a welldefined limit of algebraic varieties $\lim _{\longleftarrow} \mathfrak{g}_{n}^{\leq r}$ which we denote by $\mathfrak{s} \leq_{\infty}^{\leq r}$, where $\mathfrak{s}$ is an abbreviation for $\mathfrak{s l}, \mathfrak{s o}$ or $\mathfrak{s p}$.

The radical ideals $J_{n}^{\leq r}$ of $\mathbf{S} \cdot\left(\mathfrak{g}_{n}\right)$ with respective zero-sets $\mathfrak{g}_{n}^{\leq r} \subset \mathfrak{g}_{n}^{*}$ form a chain whose union we denote by $J \leq r$. The ideal $J \leq r$ is a radical Poisson ideal of $\mathbf{S}\left(\mathfrak{g}_{\infty}\right)$. It turns out that any non-zero radical Poisson ideal of $\mathbf{S} \cdot\left(\mathfrak{g}_{\infty}\right)$ is of this form.

Theorem 3.3. Let $\mathfrak{g}_{\infty}=\mathfrak{s l}_{\infty}, \mathfrak{s o}_{\infty}, \mathfrak{s p}_{\infty}$ and $J \subset \mathrm{S} \cdot\left(\mathfrak{g}_{\infty}\right)$ be a non-zero radical Poisson ideal. Then $J=J \leq r$ for some $r \in \mathbb{Z}_{\geq 0}$.

Theorems 3.1 and 3.3 are proved in Section 5

\section{Auxiliary Results}

Throughout the paper $V$ denotes a finite-dimensional vector space of dimension $d_{V}$. In order to consider all simple classical groups simultaneously, we use S (respectively, $\mathfrak{s}$ ) as an abbreviation for SL, SO, Sp (respectively, $\mathfrak{s l}, \mathfrak{s o}, \mathfrak{s p}$ ) and consider three different cases. In the case $\mathrm{S}=\mathrm{SL}$ we fix the zero bilinear form on the space $V$ and set $\mathrm{S}(V):=\mathrm{SL}(V), \mathfrak{s}(V):=\mathfrak{s l}(V)$. In the case $\mathrm{S}=\mathrm{SO}$ we fix a nondegenerate symmetric bilinear form on $V$ and set $\mathrm{S}(V):=\mathrm{SO}(V), \mathfrak{s}(V):=\mathfrak{s o}(V)$. In the case $\mathrm{S}=\mathrm{Sp}$ we assume that $d_{V}$ is even and fix a nondegenerate antisymmetric bilinear form on $V$. Then $\mathrm{S}(V):=\operatorname{Sp}(V), \mathfrak{s}(V):=\mathfrak{s p}(V)$.

In this section $G$ denotes a connected simple subgroup of $\mathrm{S}(V)$ with Lie algebra $\mathfrak{g} \subset \mathfrak{s}(V)$. We start with some general statements about $G$-orbits in $\mathfrak{g}^{*}$ (i.e. about coadjoint orbits). We identify $\mathfrak{g}$ and $\mathfrak{g}^{*}$ via the Cartan-Killing form. Fix $e \subset \mathfrak{g}^{*}$. Denote the $G$-orbit of $e$ in $\mathfrak{g}^{*}$ by $\mathcal{O}[e]$. Let $\underline{\mathcal{O}}[e]$ be the unique closed $G$-orbit of the closure $\overline{\mathcal{O}[e]}$ in $\mathfrak{g}^{*}$. By the Luna Slice Theorem $[\mathrm{VP}$, there exists a $G$-equivariant morphism $\mathcal{O}[e] \rightarrow \underline{\mathcal{O}}[e]$. Assume that $\underline{\mathcal{O}}[e] \neq 0$, i.e. $e$ is not nilpotent. Let $h \in \underline{\mathcal{O}}[e]$ 
be a semisimple element. Then the centralizer of $h$ in $\mathfrak{g}$ is a Levi subalgebra 1 of some parabolic subalgebra $\mathfrak{p} \subset \mathfrak{g}$. Let $P \subset G$ be a parabolic subgroup with Lie algebra $\mathfrak{p}$. Then there exists a finite $G$-equivariant covering $\tilde{\mathcal{O}} \rightarrow \mathcal{O}[e]$ and a $G$-equivariant morphism $\tilde{\mathcal{O}} \rightarrow G / P$.

Assume now that $\underline{\mathcal{O}}[e]=\{0\}$, i.e. that $e$ is nilpotent. By the Jacobson-Morozov Theorem there exist elements $h, f$ such that

$$
[h, e]=2 e,[h, f]=-2 f,[e, f]=h,
$$

i.e. such that $\{e, h, f\}$ is an $\mathfrak{s l}_{2}$-triple. The element $h$ is rational semisimple. Hence $\mathfrak{g}$ splits into the direct sum of adh-eigenspaces $\oplus_{i \in \mathbb{Q}} \mathfrak{g}_{i}$ with rational eigenvalues. The direct sum $\mathfrak{g}_{h}^{+}:=\oplus_{i \geq 0} \mathfrak{g}_{i} \subset \mathfrak{g}$ is a parabolic subalgebra of $\mathfrak{g}$ and we denote it by $\mathfrak{p}_{e}$. The subalgebra $\mathfrak{p}_{e}$ is determined by $e$. By $P_{e} \subset G$ we denote the parabolic subgroup with the Lie algebra $\mathfrak{p}_{e}$. There is a $G$-equivariant morphism $\mathcal{O}[e] \rightarrow G / P_{e}$.

The above discussion is summarized in the following lemma.

Lemma 4.1. A suitable finite covering of any coadjoint orbit admits a $G$-equivariant (and thus surjective) morphism to $G / P$, where $P$ is a maximal parabolic subgroup.

For $\mathfrak{g}=\mathfrak{s l}(V)$, a quotient $G / P$ is nothing but a Grassmannian $\operatorname{Gr}(r ; V)$ for some $r<d_{V}$. For $\mathfrak{g}=\mathfrak{s o}(V)$ and $\mathfrak{s p}(V)$ a quotient $G / P$ is an irreducible component of the variety $\operatorname{Gr}\left({ }^{0} r ; V\right)$ of isotropic subspaces in $V$ of dimension $r$ for some $r \leq \frac{d_{V}}{2}$. The variety $\operatorname{Gr}\left({ }^{0} r ; V\right)$ is irreducible unless $\mathfrak{g}=\mathfrak{s o}(V)$ and $r=\frac{d_{V}}{2}$. In this latter case $\operatorname{Gr}\left({ }^{0} r ; V\right)$ has two irreducible components which are isomorphic as varieties. More generally, for $k<r<d_{V}$, we denote by $\operatorname{Gr}\left({ }^{k} r ; V\right)$ the variety of subspaces of $V$ of dimension $r$ on which the restriction of the fixed form on $V$ has rank $k$.

For $X \in \operatorname{End} V$ we denote by $V_{\lambda}^{X} \subset V$ the generalized eigenspace of $X$ with eigenvalue $\lambda \in \mathbb{F}$. Furthermore, $\mathfrak{g} \cdot V$ stands for the sum of the non-trivial simple $\mathfrak{g}$-submodules of $V$.

Proposition 4.2. Assume that there is an $\mathrm{S}(V)$-orbit $\mathcal{O}$ in $\mathfrak{s}(V)^{*}$ such that its image in $\mathfrak{g}^{*}$ under the canonical projection $\mathfrak{s}(V)^{*} \rightarrow \mathfrak{g}^{*}$ is not dense. Then

$$
\operatorname{dim}(\mathfrak{g} \cdot V)<2(\operatorname{dim} G-\operatorname{rk} G)(\operatorname{rk} G+1) \text { or } 2 \operatorname{dim} G+2 \geq d_{V} .
$$

In order to prove Proposition 4.2 we need several preliminary statements.

Lemma 4.3. Let $V^{\prime}$ be any subspace in $V$ of dimension $d_{V^{\prime}}$.

a) Assume that $\mathfrak{s}=\mathfrak{s l}, \mathfrak{s p}$. If $\operatorname{dim}\left(V^{r} \cap V^{\prime}\right) \geq 1$ for any isotropic subspace $V^{r} \subset V$ of dimension $r$, then $r+d_{V^{\prime}}>d_{V}$.

b) Assume that $\mathfrak{s}=\mathfrak{s o}$ and $r<\frac{d_{V}}{2}$. If $\operatorname{dim}\left(V^{r} \cap V^{\prime}\right) \geq 1$ for any isotropic subspace $V^{r} \subset V$ of dimension $r$, then $r+d_{V^{\prime}}>d_{V}$.

c) Assume that $\mathfrak{s}=\mathfrak{s o}, d_{V}$ is even, and $r=\frac{d_{V}}{2}$. If $\operatorname{dim}\left(V^{r} \cap V^{\prime}\right) \geq 1$ for any isotropic subspace $V^{r} \subset V$ of dimension $r$ from some irreducible component of $\operatorname{Gr}\left({ }^{0} r ; V\right)$, then either $r+d_{V^{\prime}}>d_{V}$, or $r+d_{V^{\prime}}=d_{V}$ and $V^{\prime}$ is isotropic.

Proof. Exercise in linear algebra.

Let $Z$ be a $G$-variety. We denote the maximal dimension of a $G$-orbit on $Z$ by $\mathrm{m}_{G}(Z)$. For a subvariety $Y \subset Z$ we set

$$
G Y:=\{z \in Z \mid z=g y \text { for some } y \in Y \text { and } g \in G\} .
$$

\footnotetext{
${ }^{1}$ Under a Levi subalgebra of a parabolic subalgebra $\mathfrak{p} \subset \mathfrak{g}$ we understand a maximal reductive in $\mathfrak{g}$ subalgebra of $\mathfrak{p}$.
} 
Lemma 4.4. a) Assume that $\mathrm{m}_{G}(\mathbb{P}(V))<\operatorname{dim} G$. Then

$$
\operatorname{dim}(\mathfrak{g} \cdot V)<2(\operatorname{dim} G-\operatorname{rank} G)(\operatorname{rank} G+1) .
$$

b) Let $\mathfrak{s}=\mathfrak{s o}$. Assume that

$$
\mathrm{m}_{G}(\mathbb{P}(V))=\operatorname{dim} G \text { and } \mathrm{m}_{G}\left(\operatorname{Gr}\left({ }^{0} 1 ; V\right)\right)<\operatorname{dim} G .
$$

Then

$$
2 \operatorname{dim} G+2 \geq d_{V}
$$

Proof. The statement of part a) is the main result of [AnPo]. In part b) $\operatorname{Gr}\left({ }^{0} 1 ; V\right)=$ $\mathbb{P}(\mathcal{V})$, where $\mathcal{V}$ is the set of isotropic vectors in $V$. The inequality $\mathrm{m}_{G}(\mathbb{P}(\mathcal{V}))<\operatorname{dim} G$ implies that the stabilizer of a generic point of $\mathbb{P}(\mathcal{V})$ under the action of $G$ has positive dimension. Therefore there exists $A \in \mathfrak{g}$ such that $\mathcal{O}[A]$ intersects $\mathfrak{g}_{x}$ for all $x$ in an open subset of $\mathbb{P}(\mathcal{V})$. Then, for some eigenvalue $\lambda$ of $A$, we have $\mathcal{V} \subset \overline{G V_{\lambda}^{A}}$, where

$$
V_{\lambda}^{A}:=\operatorname{Ker}\left(A-\lambda \operatorname{Id}_{V}\right)^{d_{V}}
$$

and $\overline{G V_{\lambda}^{A}}$ is the closure of $G V_{\lambda}^{A}$ in $V$. Furthermore, the equality $\mathrm{m}_{G}(\mathbb{P}(V))=\operatorname{dim} G$ implies that $\mathcal{V}$ coincides with $\overline{G V_{\lambda}^{A}}$. Therefore $V_{\lambda}^{A} \subset \mathcal{V}$, and the space $V_{\lambda}^{A}$ is isotropic. In particular, $\operatorname{dim} V_{\lambda}^{A} \leq \frac{d_{V}}{2}$, and consequently $\operatorname{dim} G \geq \frac{d_{V}}{2}-1$.

Lemma 4.5. Let

$$
2 \leq r \leq \frac{d_{V}}{2}, \mathrm{~m}_{G}(\mathbb{P}(V))=\operatorname{dim} G \text { and } \mathrm{m}_{G}\left(\operatorname{Gr}\left({ }^{0} r ; V\right)\right)<\operatorname{dim} G
$$

for some $r$. Then

$$
2 \operatorname{dim} G+2 \geq d_{V} .
$$

Proof. Let $x \in \operatorname{Gr}\left({ }^{0} r ; V\right)$ denote a point and $V(x)$ be the corresponding $r$-dimensional space. Let $G_{x}$ be the stabilizer of $x$ in $G ; G_{x}$ acts on $V(x)$ and, if $x$ is generic, we have

$$
\mathrm{m}_{G_{x}}(\mathbb{P}(V(x)))=\operatorname{dim} G_{x} .
$$

Let $p \in V(x)$ be a non-zero vector, $\langle p\rangle \subset V(x)$ be the line generated by $p$, and $\mathfrak{g} p \subset V$ be the tangent space to $G p$ in $p$. Then $\operatorname{dim}((V(x) /\langle p\rangle) \cap \mathfrak{g}\langle p\rangle) \geq \operatorname{dim} G_{x}$, where $\mathfrak{g}\langle p\rangle$ is the image of $\mathfrak{g} p$ in $V /\langle p\rangle$. Hence, for a generic $\langle p\rangle \in \operatorname{Gr}\left({ }^{0} 1 ; V\right)$ and any

$$
\tilde{V} \in \operatorname{Gr}\left({ }^{0}(r-1) ;\langle p\rangle^{\perp} /\langle p\rangle\right)
$$

we have $\operatorname{dim}(\tilde{V} \cap \mathfrak{g}\langle p\rangle) \geq \operatorname{dim} G_{x}$. In particular, $\operatorname{dim}(\tilde{V} \cap \mathfrak{g}\langle p\rangle) \geq 1$. Therefore, by Lemma 4.3 (applied to $V^{\prime}=\mathfrak{g}\langle p\rangle$ and $V=\langle p\rangle^{\perp} /\langle p\rangle$ ) we obtain

$$
r-1+\operatorname{dimg} \geq \operatorname{dim}\left(\langle p\rangle^{\perp} /\langle p\rangle\right) .
$$

As $\operatorname{dim}\left(\langle p\rangle^{\perp} /\langle p\rangle\right) \geq d_{V}-2$ and $r \leq \frac{d_{V}}{2}$, we conclude that $2 \operatorname{dim} G+2 \geq d_{V}$.

Proposition 4.6 ( $\mathrm{Vi}$ text after Prop. 3]). Let $Z$ be an irreducible $G$-symplectic variety with a moment map $\Phi: Z \rightarrow \mathfrak{g}^{*}$. Then the dimension of $\Phi(Z)$ equals $\mathrm{m}_{G}(Z)$.

Proof of Proposition 4.2. Since we can consider the morphism $\mathcal{O} \rightarrow \mathfrak{g}^{*}$ as a moment map, Proposition 4.6 implies that the image of $\mathcal{O}$ in $\mathfrak{g}^{*}$ is not dense if and only if

$$
\mathrm{m}_{G}(\mathcal{O})<\operatorname{dim} G \text {. }
$$

Furthermore, according to Lemma 4.1, for some $r, 1 \leq r \leq \frac{d_{V}}{2}$, there exists an $\mathrm{S}(V)$-equivariant morphism from a finite covering of $\mathcal{O}$ onto $\operatorname{Gr}\left({ }^{0} r ; V\right)$. Therefore 


$$
\mathrm{m}_{G}\left(\operatorname{Gr}\left({ }^{0} r ; V\right)\right)<\operatorname{dim} G .
$$

If $\mathrm{m}_{G}\left(\operatorname{Gr}\left({ }^{0} 1 ; V\right)\right)<\operatorname{dim} G$, the claim of Proposition 4.2 follows from Lemma 4.4 Indeed, if also $\mathrm{m}_{G}(\mathbb{P}(V))<\operatorname{dim} G$, we apply Lemma 4.4 a), and if $\mathrm{m}_{G}(\mathbb{P}(V))=$ $\operatorname{dim} G$, then $\mathfrak{s}=\mathfrak{s o}\left(\right.$ as $\left.\mathbb{P}(V) \neq \operatorname{Gr}\left({ }^{0} 1 ; V\right)\right)$ and we apply Lemma $\left.4.4 \mathrm{~b}\right)$.

If

$$
\mathrm{m}_{G}\left(\operatorname{Gr}\left({ }^{0} 1 ; V\right)\right)=\operatorname{dim} G,
$$

then necessarily $\mathrm{m}_{G}(\mathbb{P}(V))=\operatorname{dim} G$ and $r \geq 2$. In this case the claim of Proposition 4.2 follows from Lemma 4.5 .

Proposition 4.2 is used in a crucial way in the proof of Theorem 3.1. The results in the remaining part of this section are necessary for the proof of Theorem 3.3

In what follows $W$ denotes a subspace of $V$ of dimension $d_{W}$. If $\mathfrak{s}=\mathfrak{s o}, \mathfrak{s p}$, we assume that the restriction of the fixed form on $V$ is nondegenerate on $W$. This yields embeddings $\mathrm{S}(W) \rightarrow \mathrm{S}(V)$ and $\mathfrak{s}(W) \rightarrow \mathfrak{s}(V)$. For $\mathfrak{s}=\mathfrak{s l}$ an embedding $\mathrm{S}(W) \rightarrow \mathrm{S}(V)$ is determined by a choice of complement to $W$ in $V$. Moreover, in all three cases we have an orthogonal (with respect to the Cartan-Killing form on $\mathfrak{s}(V))$ projection $\phi: \mathfrak{s}(V) \rightarrow \mathfrak{s}(W)$. It is easy to see that $\phi(X)=\operatorname{pr}_{W} \circ\left(\left.X\right|_{W}\right)$, where $X \in \mathfrak{s}(V)$ is viewed as an element of End $V$ and $\operatorname{pr}_{W}: V \rightarrow W$ is the orthogonal projection for $\mathfrak{s}=\mathfrak{s o}, \mathfrak{s p}$, and respectively the projection along the fixed complement of $W$ in $V$ for $\mathfrak{s}=\mathfrak{s l}$. We fix the embedding $\mathfrak{s}(W) \rightarrow \mathfrak{s}(V)$, and $\phi: \mathfrak{s}(V) \rightarrow \mathfrak{s}(W)$ stands for the corresponding projection.

Recall that $\mathcal{O}[X]$ denotes the $\mathrm{S}(V)$-orbit in $\mathfrak{s}(V)$ of $X \in \mathfrak{s}(V)$.

Lemma 4.7. Let $X \in \mathfrak{s}(V)$. Then

a) $\operatorname{rk} \phi(X) \leq \operatorname{rk} X$

b) if $d_{W}>\operatorname{rk} X$, the image $\phi(\mathcal{O}[X])$ contains an element of $\operatorname{rank} \operatorname{rk} X$.

Proof. Part a) follows immediately from the formula

$$
\phi(X)=\operatorname{pr}_{W} \circ\left(\left.X\right|_{W}\right) .
$$

To prove part b) we consider a generic subspace $\tilde{W} \subset W$ of dimension $d_{W}$. Note that for $\mathfrak{s}=\mathfrak{s o}, \mathfrak{s p}$ the restriction of the form to $\tilde{W}$ is nondegenerate. Furthermore, $\left.\operatorname{rk} X\right|_{\tilde{W}}=\operatorname{rk} X$, and $\tilde{W}^{\perp} \cap \operatorname{Im} X=0$ where $\operatorname{Im} X$ is the image of $X \in \operatorname{End} V$. Therefore $\operatorname{rk}\left(\left.\operatorname{pr}_{\tilde{W}} \circ X\right|_{\tilde{W}}\right)=\operatorname{rk} X$.

Since there exists $g \in \mathrm{S}(V)$ with $g(W)=\tilde{W}$, we have

$$
\operatorname{rk} \phi(g(X))=\operatorname{rk} X \text {. }
$$

Proof. The proof is very similar to the proof of Lemma 4.7

Lemma 4.8. Assume $r \leq \frac{d_{W}}{2}$. Then the set $\mathfrak{s}(V)^{\leq r}$ defined by (2) is the largest $\mathrm{S}(V)$-invariant subset of the preimage $\phi^{-1}\left(\mathfrak{s}(W)^{\leq r}\right)$.

Proof. Exercise in linear algebra.

Lemma 4.9. Consider the projection $\varphi: \mathfrak{s p}(V)^{*} \rightarrow \mathfrak{g l}\left(V^{i s o}\right)^{*}$ dual to the embed$\operatorname{ding} \mathfrak{g l}\left(V^{\text {iso }}\right) \rightarrow \mathfrak{s p}(V)$, where $V^{\text {iso }}$ is a maximal isotropic subspace of $V$. Assume $r \leq \frac{d_{V}}{2}$. Then the set $\mathfrak{s p}(V) \leq r$ defined by $(2)$ is the largest $\operatorname{Sp}(V)$-invariant subset of the preimage $\varphi^{-1}\left(\mathfrak{s l}\left(V^{\text {iso }}\right) \leq r\right)$. 
Proof. Exercise in linear algebra.

Lemma 4.10. Let $x \in \operatorname{Gr}(r ; V)$ and let $V(x) \subset V$ be the corresponding subspace. Let $W^{\prime}$ be the $\mathrm{S}(W)$-stable complement to $W$ in $V$. Assume that $d_{V} \geq 2 d_{W}$, $d_{W} \leq r \leq d_{V}-d_{W}$, and $\operatorname{dim}\left(V(x) \cap W^{\prime}\right)=r-d_{W}$. Then the stabilizer of $x \in \operatorname{Gr}(r ; V)$ in $\mathrm{SL}(W)$ (and therefore also in $\mathrm{SO}(W), \mathrm{Sp}(W)$ ) is trivial.

Proof. Exercise in linear algebra.

For a subset $S \subset \mathbb{F}$ we set $V_{S}^{X}:=\oplus_{\lambda \in S} V_{\lambda}^{X}$. We set also $\hat{0}:=\mathbb{F} \backslash\{0\}$; then $V=V_{0}^{X} \oplus V_{\hat{0}}^{X}$. Let $X_{n n}:=\left.X\right|_{V_{0}}, X_{r}:=\left.X\right|_{V_{\hat{0}}}$, and $X_{r}=X_{s}+X_{n s}$ be the Jordan decomposition as sum of commuting semisimple and nilpotent elements. The decomposition $V=V_{0}^{X} \oplus V_{\hat{0}}^{X}$ allows as to consider all four operators $X_{n n}, X_{r}, X_{s}, X_{n s}$ as endomorphisms of $V$. Furthermore,

and $X_{s}+X_{n n} \in \overline{\mathcal{O}[X]}$.

$$
\operatorname{rk}\left(X_{s}+X_{n s}\right)=\operatorname{rk} X_{s}, \quad \operatorname{rk}\left(X_{s}+X_{n n}\right)=\operatorname{rk} X
$$

Definition 4.11. We say that $X \in \operatorname{End} V$ is rank-reduced whenever

$$
X_{n n}^{2}=0 \text { and } X_{n s}=0 .
$$

Lemma 4.12. If for some $X \in \mathfrak{s}(V)$ we have $2 \operatorname{rk} X \leq d_{V}$, then there exists a (unique up to conjugation) rank-reduced element $X^{\prime} \in \mathfrak{s}(V)$ such that $X^{\prime} \subset \overline{\mathcal{O}[X]}$ and $\operatorname{rk} X=\operatorname{rk} X^{\prime}$.

Proof. The condition $\left(X_{n n}^{\prime}\right)^{2}=0$ means that $X_{n n}^{\prime}$ is nilpotent and the sizes of its Jordan blocks are at most $2 \times 2$. Moreover, the number of non-zero Jordan blocks of $X_{n n}^{\prime}$ equals rk $X_{n n}^{\prime}$. In particular, all nilpotent rank-reduced endomorphisms in $\mathfrak{s}(V)$ of fixed rank are conjugate (possibly, by outer automorphisms of $\mathfrak{s}(V)$ ). Since $2 \operatorname{rk} X_{n n} \leq d_{V}$, there exists a nilpotent rank-reduced endomorphism $X_{r r}$ of rank equal to rk $X$ and such that $X_{r r} \in \overline{\mathcal{O}\left[X_{n n}\right]}$ [CM]. A rank-reduced endomorphism $X^{\prime} \in \mathfrak{s}(V)$ such that $X_{s}^{\prime}=X_{s}$ and $X_{n n}^{\prime}$ is conjugate to $X_{r r}$, is as desired. By construction, $X^{\prime}$ is unique up to conjugation.

Lemma 4.13. Assume $d_{V}>3 d_{W}$. If for some $X \in \mathfrak{s}(V)$ the image $\phi(\mathcal{O}[X])$ is not dense in $\mathfrak{s}(W)$, then there exists a unique $\lambda \in \mathbb{F}$ such that

$$
\operatorname{rk}\left(X-\lambda \operatorname{Id}_{V}\right)<d_{W} \text {. }
$$

Proof. Set $d_{V}(\mu):=\operatorname{dim} V_{\mu}^{X}$ and $d_{V}(S):=\sum_{\mu \in S} d_{V}(\mu)$ for any subset $S \subset \mathbb{F}$. We have an $\mathrm{S}(V)$-equivariant morphism

$$
\mathcal{O}[X] \rightarrow \operatorname{Gr}\left(d_{V}(S) ; V\right), \quad X \longmapsto \oplus_{\mu \in S} V_{\mu}^{X} .
$$

As $\phi(\mathcal{O}[X])$ is not dense in $\mathfrak{s}(W)$, the inequality $\mathrm{m}_{\mathrm{S}(W)}(\mathcal{O}[X])<\operatorname{dim} \mathrm{S}(W)$ holds. Therefore, for any $S \subset \mathbb{F}$, there exists $k$ such that

$$
\mathrm{m}_{\mathrm{S}(W)}\left(\operatorname{Gr}\left({ }^{k} d_{V}(S) ; V\right)\right)<\operatorname{dim} \mathrm{S}(W) .
$$

We now show that, for any $S \subset \mathbb{F}$, either $d_{V}(S)<d_{W}$ or $d_{V}(S)>d_{V}-d_{W}$. Assume to the contrary that $d_{W} \leq d_{V}(S) \leq d_{V}-d_{W}$. Let $x \in \operatorname{Gr}\left({ }^{k} d_{V}(S) ; V\right)$ be a generic point and $V(x) \subset V$ be the corresponding subspace. Then

$$
\operatorname{dim}\left(V(x) \cap W^{\prime}\right)=\operatorname{dim} V(x)-d_{W},
$$


where $W^{\prime}$ is the $\mathrm{S}(W)$-stable complement to $W$ in $V$, and Lemma4.10 implies that the stabilizer of $x$ in $\mathrm{S}(W)$ is trivial. This contradicts (3).

We claim next that there exists $\lambda \in \mathbb{F}$ such that $d_{V}(\lambda)>d_{V}-d_{W}$. Indeed, assuming that $d_{V}(\lambda)<d_{W}$ for all $\lambda \in \mathbb{F}$, we see that the inequality $3 d_{W}<d_{V}$, together with the alternative $d_{V}(S)<d_{W}$ or $d_{V}(S)>d_{V}-d_{W}$, implies $d_{V}(S)<d_{W}$ for any finite set $S$. This is again a contradiction.

Fix now $\lambda$ with $d_{V}(\lambda)>d_{V}-d_{W}$. It is easy to see that $\operatorname{rk}\left(X-\lambda \operatorname{Id}_{V}\right)=$ $d_{V}(\mathbb{C} \backslash \lambda)+\left.\operatorname{rk}\left(X-\lambda \operatorname{Id}_{V}\right)\right|_{V_{\lambda}^{X}}$. Furthermore, we have a decomposition

$$
X-\lambda \operatorname{Id}_{V}=\left(X-\lambda \operatorname{Id}_{V}\right)_{s}+\left(X-\lambda \operatorname{Id}_{V}\right)_{n s}+\left(X-\lambda \operatorname{Id}_{V}\right)_{n n} .
$$

Let $r^{\prime} \in \mathbb{Z}_{\geq 0}$ for $\mathfrak{s}=\mathfrak{s l}$ and $r^{\prime} \in 2 \mathbb{Z}_{\geq 0}$ for $\mathfrak{s}=\mathfrak{s o}, \mathfrak{s p}$. If $r^{\prime}<\operatorname{rk}\left(\left.\left(X-\lambda \operatorname{Id}_{V}\right)_{n n}\right|_{V_{\lambda}^{X}}\right)$, there exists $X_{r^{\prime}}^{\lambda} \in \overline{\mathcal{O}\left[\left.\left(X-\lambda \operatorname{Id}_{V}\right)_{n n}\right|_{V_{\lambda}^{X}}\right]} \subset \mathfrak{s}\left(V_{\lambda}^{X}\right)$ with $\operatorname{rk} X_{r^{\prime}}^{\lambda}=r^{\prime}$. Hence, if $\operatorname{rk}\left(\left(X-\lambda \operatorname{Id}_{V}\right)_{s}\right) \leq r^{\prime} \leq \operatorname{rk}\left(X-\lambda \operatorname{Id}_{V}\right)$, there exists

$$
X_{r^{\prime}} \in \overline{\mathcal{O}[X]} \subset \mathfrak{s}(V)
$$

with $X_{r^{\prime}}-\lambda \mathrm{Id}=(X-\lambda \mathrm{Id})_{s}+X_{r^{\prime}-\operatorname{rk}\left((X-\lambda \mathrm{Id})_{s}\right)}^{\lambda}$, and thus $\operatorname{rk}\left(X_{r^{\prime}}-\lambda \mathrm{Id}_{V}\right)=r^{\prime}$.

As, for any $d$ with $d_{W} \leq d \leq d_{V}-d_{W}$, the equality $\mathrm{m}_{\mathrm{S}(W)}\left(\operatorname{Gr}\left({ }^{k} d ; V\right)\right)=\operatorname{dim} \mathrm{S}(W)$ holds for some $k$, for any $r^{\prime}$ we have either $\operatorname{dim} \operatorname{Im}\left(X_{r^{\prime}}-\lambda \operatorname{Id}_{V}\right)<d_{W}$ or $\operatorname{dim} \operatorname{Im}\left(X_{r^{\prime}}-\right.$ $\left.\lambda \operatorname{Id}_{V}\right)>d_{V}-d_{W}$. Therefore, either $\operatorname{rk}\left(X-\lambda \operatorname{Id}_{V}\right)<d_{W}$ or $\operatorname{rk}\left(X-\lambda \operatorname{Id}_{V}\right)_{s}>$ $d_{V}-d_{W}>d_{W}$. On the other hand, $\operatorname{rk}\left(X-\lambda \operatorname{Id}_{V}\right)_{s}<d_{W}$. This implies

$$
\operatorname{rk}\left(X-\lambda \operatorname{Id}_{V}\right)<d_{W} .
$$

Lemma 4.14. Assume $d_{V} \geq 2 d_{W}, d_{W}>r$, and let $X \in \mathfrak{s}(V)^{\leq r}$. If $\operatorname{rk}\left(X-\lambda \operatorname{Id}_{V}\right)=$ $r$ for some $\lambda \in \mathbb{F}$, then $\phi(\mathcal{O}[X])$ is dense in $\mathfrak{s}(W)^{\leq r}$.

We consider separately the cases $\mathfrak{s}=\mathfrak{s l}$ and $\mathfrak{s}=\mathfrak{s o}, \mathfrak{s p}$.

Proof of Lemma 4.14 for $\mathfrak{s}=\mathfrak{s l}$. By Lemma 4.12 we can assume that $X-\lambda \operatorname{Id}_{V}$ is rank-reduced. Therefore the linear operator $X-\lambda \operatorname{Id}_{V}: V \rightarrow V$ is conjugate to a direct sum

$$
\bigoplus_{1 \leq i \leq \mathrm{rk} X_{s}} A\left(t_{i}\right) \bigoplus_{1 \leq j \leq \mathrm{rk} X_{n n}} B \bigoplus C
$$

where $C$ is the zero operator, and $A(t), B$ are operators of the following forms:

$$
A(t):=\left(\begin{array}{cc}
t & 0 \\
0 & 0
\end{array}\right), \quad B:=\left(\begin{array}{cc}
0 & 1 \\
0 & 0
\end{array}\right) .
$$

We denote the elements of the respective bases of the 2-dimensional subspaces of $V$ corresponding to $A\left(t_{i}\right)$ and $B$ by $a_{1}[i], a_{2}[i]$ and $b_{1}[j], b_{2}[j]$, and the elements of the basis of the subspace corresponding to $C$ by $c[k]$. We can further assume that

$$
W:=\operatorname{span}\left\{a_{1}[i], b_{1}[j], c[k] \mid 1 \leq i \leq \operatorname{rk} X_{s}, 1 \leq j \leq \operatorname{rk} X_{n n}, 1 \leq k \leq d_{W}-r\right\}
$$

and that the $\mathrm{SL}(W)$-invariant complement $W^{\prime}$ of $W$ is the span of the remaining elements of the basis $\left\{a_{1}[i], a_{2}[i], b_{1}[j], b_{2}[j], c_{k}\right\}_{i, j, k}$ of $V$. Then

$\phi\left(\bigoplus_{i} \mathcal{O}\left[A\left(t_{i}\right)\right] \bigoplus_{j} \mathcal{O}[B] \bigoplus C\right) \subset \mathfrak{s l}\left(\bigoplus_{1 \leq i \leq \mathrm{rk} X_{s}} \mathfrak{g l}\left\langle a_{1}[i], a_{2}[i]\right\rangle \bigoplus_{1 \leq j \leq \mathrm{rk} X_{n n}} \mathfrak{s l}\left\langle b_{1}[i], b_{2}[i]\right\rangle\right)$.

Moreover, $\phi\left(\bigoplus_{i} \mathcal{O}\left[A\left(t_{i}\right)\right] \bigoplus_{j} \mathcal{O}[B] \bigoplus C\right)$ consists of all diagonal matrices of the form 


$$
\operatorname{diag}\left(\lambda_{1}, \ldots, \lambda_{r}, 0, \ldots, 0\right), \quad \lambda_{1}+\ldots+\lambda_{r}=0 .
$$

This follows from the obvious statement that the matrices in $\mathcal{O}[A(t)]$ for $t \neq 0$, and in $\mathcal{O}[B]$ can have arbitrary values in their left top corner. Therefore $\phi(\mathcal{O}[X])$ contains all semisimple elements of a given $\operatorname{rank} r=\operatorname{rk} X$. As such elements are dense in $\mathfrak{s l}(W)^{\leq r}, \phi(\mathcal{O}[X])$ is dense in $\mathfrak{s l}(W)^{\leq r}$.

In the rest of this section $\mathfrak{s}=\mathfrak{s o}, \mathfrak{s p}$. To prove Lemma 4.14 in this case, we need some preliminary lemmas.

Lemma 4.15. Let $X \in \mathfrak{s}(V)$. Assume that $2 \operatorname{rk}\left(X-\lambda \operatorname{Id}_{V}\right)<d_{V}$ for some $\lambda \in \mathbb{F}$. Then $\lambda=0$.

Proof. Obviously, $\operatorname{rk}\left(X-\lambda \operatorname{Id}_{V}\right)+d_{V}(\lambda) \geq d_{V}$ (recall that $\left.d_{V}(\lambda)=\operatorname{dim} V_{\lambda}^{X}\right)$. Therefore $2 \operatorname{rk}\left(X-\lambda \operatorname{Id}_{V}\right)<d_{V}$ implies that $2 d_{V}(\lambda)>d_{V}$.

However, for $\mathfrak{s o}, \mathfrak{s p}$, we have $d_{V}(\lambda)=d_{V}(-\lambda)$. Therefore $\lambda \neq 0$ would mean that $\lambda \neq-\lambda$ and $\operatorname{dim} V_{\lambda}^{X}+\operatorname{dim} V_{-\lambda}^{X}>d_{V}$, which is false. Hence $\lambda=0$.

Fix $r \in \mathbb{Z}_{\geq 0}$. If $r$ is even we denote by $\mathfrak{s}(V)_{\text {reg }}^{r}$ the set of semisimple elements of $\mathfrak{s}(V)$ which have rank $r$. If $r$ is odd we denote by $\mathfrak{s p}(V)_{\text {reg }}^{r}$ the set of elements $X$ of $\mathfrak{s p}(V)$ such that

$$
\operatorname{rk} X_{s}=r-1, X_{n s}=0, \operatorname{rk} X_{n n}=1 .
$$

In particular, $X_{n n}$ is nilpotent and has a single non-zero Jordan block of size $2 \times 2$.

Lemma 4.16. a) Assume $d_{V}$ is even. Then $\overline{\mathfrak{s p}(V)_{\text {reg }}^{d_{V}-1}}=\mathfrak{s p}(V) \leq\left(d_{V}-1\right)$.

b) Assume $d_{V}$ is odd. Then $\overline{\mathfrak{s o}(V)_{\text {reg }}^{d_{V}-1}}=\mathfrak{s o}(V) \leq\left(d_{V}-1\right)$.

Proof. Exercise in linear algebra.

In connection with the following two lemmas, note that $\operatorname{rk} X$ is even for any $X \in \mathfrak{s o}(V)$. Recall also that on $\mathfrak{s}(V)$ there is the following partial order: $X_{1}$ is lower than $X_{2}$ if $\mathcal{O}\left[X_{1}\right] \subset \overline{\mathcal{O}\left[X_{2}\right]}$. This order can be described explicitly in terms of Young diagrams (or equivalently, in terms of the Jordan normal forms of $X_{1}, X_{2}$ ), see $\mathrm{CM}$.

Lemma 4.17. Let $r$ be an odd integer such that $1 \leq r \leq d_{V}$; assume that $d_{V}$ is even. Then $\mathfrak{s p}(V) \leq r=\overline{\mathfrak{s p}(V)_{\text {reg }}^{r}}$.

Proof. By definition $\mathfrak{s p}(V)_{\text {reg }}^{r} \subset \mathfrak{s p}(V) \leq r$. Therefore is sufficient to prove that any element $X$ of rank at most $r$ lies in $\overline{\mathfrak{s p}(V)_{\text {reg }}^{r}}$. Fix $X \in \mathfrak{s p}(V) \leq r$. There exists an $X$-stable orthogonal decomposition $V_{s} \oplus V_{n}=V$ such that $\left.X\right|_{V_{n}}$ is nilpotent and $\left.X\right|_{V_{s}}$ is given (in an appropriate basis) by a matrix of type

$$
A:=\left(\begin{array}{cc}
F & 0 \\
0 & -F^{t}
\end{array}\right) .
$$

Set $r_{s}:=\left.\operatorname{rk} X\right|_{V_{s}}, r_{n}:=\left.\operatorname{rk} X\right|_{V_{n}}$. Then $r_{s}$ and $d_{V_{s}}:=\operatorname{dim} V_{s}$ are even, and $r_{s}+r_{n}=r$.

To prove that $X \in \overline{\mathfrak{s p}(V)_{\text {reg }}^{r}}$ it suffices to prove that $\left.X\right|_{V_{s}} \in \mathfrak{s p}(V)_{\text {reg }}^{r_{s}}$ and $\left.X\right|_{V_{n}} \in \overline{\mathfrak{s p}\left(V_{n}\right)_{\text {reg }}^{r_{n}}}$. First of all we check that $\left.X\right|_{V_{s}} \in \overline{\mathfrak{s p}\left(V_{s}\right)_{\text {reg }}^{r_{s}}}$. The matrices of type $A$ form a subalgebra isomorphic to $\mathfrak{g l}_{m}$ for $m=\frac{d_{V_{s}}}{2}$. As $\left.X\right|_{V_{s}} \in \mathfrak{g l}_{m}^{\leq \frac{r_{s}}{2}}$, we have $\left.X\right|_{V_{s}} \in \overline{\left(\mathfrak{g l}_{m}\right)_{s s}^{\frac{r_{s}}{2}}}$. Therefore $\left.X\right|_{V_{s}} \in \overline{\mathfrak{s p}\left(V_{s}\right)_{\text {reg }}^{r_{s}}}$. 
Set $X^{\prime}:=\left.X\right|_{V_{n}}$. It remains to show that $X^{\prime} \in \overline{\mathfrak{s p}\left(V_{n}\right)_{r e g}^{r_{n}}}$ (note that $\operatorname{rk} X^{\prime}=r_{n}$ ).

Let $X_{\max } \in \mathfrak{s p}\left(V_{n}\right)$ be a nilpotent element of rank $r_{n}$ with a single non-zero Jordan block of size $\left(r_{n}+1\right) \times\left(r_{n}+1\right)$. Such an element of $\mathfrak{s p}\left(V_{n}\right)$ exists by [CM], and moreover, $X^{\prime} \in \overline{\mathcal{O}\left[X_{\max }\right]}$.

It suffices to prove that $X_{\max } \in \overline{\mathfrak{s p}\left(V_{n}\right)_{\text {reg }}^{r_{n}}}$. Using the explicit description of the coadjoint orbits of a simplectic group given in [CM] one can check that there exists an $X_{\text {max }}$-stable orthogonal decomposition $V_{n}^{\prime} \oplus V_{n}^{\prime \prime}=V$ such that

$$
\left.X_{\max }\right|_{V_{n}^{\prime \prime}}=0, d_{V_{n}^{\prime}}=\operatorname{rk} X_{\max }+1 .
$$

By Lemma 4.16, $\left.X_{\max }\right|_{V_{n}^{\prime}} \in \mathfrak{s p}\left(V_{n}^{\prime}\right)_{r e g}^{r_{n}}$, and thus $X_{\max } \in \overline{\mathfrak{s p}\left(V_{n}\right)_{r e g}^{r_{n}}}$.

Lemma 4.18. Let $r$ be an even integer such that $2 \leq r<d_{V}$. Then

$$
\mathfrak{s}(V)^{\leq r}=\overline{\mathfrak{s}(V)_{\text {reg }}^{r}} \text {. }
$$

Proof. By definition, $\mathfrak{s}(V)_{\text {reg }}^{r} \subset \mathfrak{s}(V) \leq r$. Therefore its suffices to prove that any element $X$ of rank at most $r$ lies in $\overline{\mathfrak{s}(V)_{\text {reg }}^{r}}$. For $\mathfrak{s}=\mathfrak{s o}$ the arguments are the same as in the proof of Lemma 4.17. This applies also to the case $\mathfrak{s}=\mathfrak{s p}$ if $\operatorname{rk} X$ is odd. Thus in the remainder of the proof we can assume that $\mathfrak{s}=\mathfrak{s p}$ and $\operatorname{rk} X$ is even.

Let $X_{\max } \in \mathfrak{s p}\left(V_{n}\right)$ be a nilpotent element of rank $r_{n}$ with two non-zero Jordan block of sizes $r_{n} \times r_{n}$ and $2 \times 2$. Such an element of $\mathfrak{s p}\left(V_{n}\right)$ exists by [CM], and moreover, $X^{\prime} \in \overline{\mathcal{O}\left[X_{\max }\right]}$.

It suffices to prove that $X_{\max } \in \overline{\mathfrak{s p}(V)_{\text {reg }}^{r}}$. There exists an $X_{\text {max }}$-stable orthogonal decomposition $V_{n}^{\prime} \oplus V_{n}^{\prime \prime} \oplus V_{n}^{\prime \prime \prime}=V$ such that

$$
d_{V_{n}^{\prime \prime}}=2, d_{V_{n}^{\prime}}=\operatorname{rk} X_{\max } \text {, and }\left.X\right|_{V_{n}^{\prime \prime \prime}}=0 .
$$

Since $\left.X\right|_{V_{n}^{\prime}} \in \overline{\mathfrak{s p}\left(V_{n}^{\prime}\right)_{r e g}^{d_{V_{n}^{\prime}}-1}},\left.X\right|_{V_{n}^{\prime} \oplus V_{n}^{\prime \prime}}$ lies in the closure in $\mathfrak{s p}\left(V_{n}^{\prime} \oplus V_{n}^{\prime \prime}\right)$ of the set of elements $Y \in \mathfrak{s p}\left(V_{n}^{\prime} \oplus V_{n}^{\prime \prime}\right) \leq r$ such that

$$
\operatorname{rk} Y_{s s}=\operatorname{rk} X_{\max }-2, \operatorname{rk} Y_{n n}=2 .
$$

As $Y_{n n} \in \overline{\mathfrak{s p}\left(\mathbb{F}^{4}\right)_{\text {reg }}^{2}}$, we have $X_{\text {max }} \in \overline{\mathfrak{s p}(V)_{\text {reg }}^{r}}$.

Proof of Lemma 4.14 for $\mathfrak{s}=\mathfrak{s o}, \mathfrak{s p}$. By Lemma 4.15, we have $\lambda=0$, and therefore $\operatorname{rk} X=r$. By Lemma 4.12 we can assume that $X$ is rank-reduced. Note that the standard $\mathfrak{g l}_{2}$-subalgebra of $\mathfrak{s}_{4} \cong \mathfrak{s o}_{4}, \mathfrak{s p}_{4}$ given by matrices

$$
\left(\begin{array}{cc}
F & 0 \\
0 & -F^{t}
\end{array}\right), \quad F \in \mathfrak{g l}_{2}
$$

in an appropriate basis.

Assume that rk $X$ is even. Then $X$ is conjugate (via $\mathrm{S}(V)$ ) to a direct sum

$$
\bigoplus_{1 \leq i \leq \mathrm{rk} X_{s}} A\left(t_{i}\right) \bigoplus_{1 \leq j \leq \mathrm{rk} X_{n n}} B \oplus C
$$

where $C$ is the zero-operator, and $A(t), B$ are of the form

$$
A(t):=\left(\begin{array}{cccc}
t & 0 & 0 & 0 \\
0 & 0 & 0 & 0 \\
0 & 0 & -t & 0 \\
0 & 0 & 0 & 0
\end{array}\right), \quad B:=\left(\begin{array}{cccc}
0 & 1 & 0 & 0 \\
0 & 0 & 0 & 0 \\
0 & 0 & 0 & 0 \\
0 & 0 & -1 & 0
\end{array}\right) .
$$


Let $a_{1}[i], a_{2}[i], a_{3}[i], a_{4}[i]$ and $b_{1}[j], b_{2}[j], b_{3}[j], b_{4}[j]$ denote the elements of the basis of the 4-dimensional subspaces of $V$ corresponding to $A\left(t_{i}\right)$ and $B$ respectively, and let $c[k]$ be the elements of the basis of the subspace corresponding to $C$. The restriction of the fixed form to

$$
\begin{gathered}
E:=\operatorname{span}\left\{a_{1}[i], a_{3}[i], b_{1}[j], b_{3}[i], c[k] \mid\right. \\
\left.1 \leq i \leq \operatorname{rk} X_{s}, 1 \leq j \leq \operatorname{rk} X_{n n}, 1 \leq k \leq d_{W}-2 r\right\}
\end{gathered}
$$

is nondegenerate and therefore we can assume that $W=E$. The orthogonal complement $W^{\perp}$ to $W$ is the span of the remaining elements in the above basis of $V$. Then

$$
\phi\left(\bigoplus_{i} \mathcal{O}\left[A\left(t_{i}\right)\right] \bigoplus_{j} \mathcal{O}[B] \bigoplus C\right) \subset \bigoplus_{1 \leq i \leq \mathrm{rk} X_{s}} \mathfrak{g l}_{2} \bigoplus_{1 \leq j \leq \mathrm{rk} X_{n n}} \mathfrak{g l}_{2} \subset \mathfrak{s}(V),
$$

and we claim that $\phi\left(\bigoplus_{i} \mathcal{O}\left[A\left(t_{i}\right)\right] \bigoplus_{j} \mathcal{O}[B] \bigoplus C\right)$ contains all diagonal matrices of the form

$$
\operatorname{diag}\left(\lambda_{1},-\lambda_{1}, \lambda_{2},-\lambda_{2} \ldots, \lambda_{\operatorname{rk} X},-\lambda_{\operatorname{rk} X}, 0, \ldots, 0\right) .
$$

This follows from the obvious statement that matrices in $\mathcal{O}[A(t)]$ for $t \neq 0$ and in $\mathcal{O}[B]$ can have arbitrary values in their left top $2 \times 2$-corner for $\mathfrak{s}=\mathfrak{s p}$ and arbitrary antisymmetric values for $\mathfrak{s}=\mathfrak{s o}$.

Therefore $\phi(\mathcal{O}[X])$ contains all semisimple elements of rank $r=\operatorname{rk} X$. As such elements are dense in $\mathfrak{s}(W)^{\leq r}$ for $r$ even, $\phi(\mathcal{O}[X])$ is dense in $\mathfrak{s}(W)^{\leq r}$.

It remains to consider the case when $\operatorname{rk} X$ is odd. Here $\mathfrak{s}=\mathfrak{s p}$. Arguments similar to those above show that $\phi(\mathcal{O}[X])$ contains all elements of $\mathfrak{s p}(W)$ which has the form

$$
\operatorname{diag}\left(\lambda_{1},-\lambda_{1}, \lambda_{2},-\lambda_{2}, \ldots, \lambda_{\frac{r-1}{2}},-\lambda_{\frac{r-1}{2}}, 0, \ldots, 0\right) \oplus\left(\begin{array}{cc}
0 & 1 \\
0 & 0
\end{array}\right)
$$

in an appropriate basis. Then $\phi(\mathcal{O}[X])$ is dense in $\mathfrak{s p}(W)^{\leq r}$ by Lemma 4.17 .

\section{Proof of Theorems 3.1 and 3.3}

In the rest of the paper we assume that $\mathfrak{g}_{\infty}$ is a locally simple Lie algebra which may be finitary (i.e. isomorphic to $\mathfrak{s l}_{\infty}, \mathfrak{s o}_{\infty}, \mathfrak{s p}_{\infty}$ ), diagonal, or non-diagonal. Let $\mathfrak{g}_{\infty}=\lim _{\rightarrow} \mathfrak{g}_{n}$ for a fixed sequence of embeddings (1). In the special case of $\mathfrak{g}_{\infty} \cong \mathfrak{s l}_{\infty}, \mathfrak{s o}_{\infty}, \mathfrak{s p}_{\infty}$ we assume in addition that the simple Lie algebras $\mathfrak{g}_{n}$ satisfy the assumptions from Section 3. Let $G_{n}$ be the adjoint group of $\mathfrak{g}_{n}$. Recall that, if $J \subset \mathbf{S}(\mathfrak{g})$ is a Poisson ideal of locally infinite codimension, the intersections $J_{n}:=$ $J \cap \mathbf{S}^{\cdot}\left(\mathfrak{g}_{n}\right)$ determine proper $G_{n}$-stable closed subvarieties $\operatorname{Var}\left(J_{n}\right) \subset \mathfrak{g}_{n}^{*}$ which form an inverse system

$$
\ldots \rightarrow \operatorname{Var}\left(J_{n}\right) \stackrel{p_{n}}{\longrightarrow} \operatorname{Var}\left(J_{n-1}\right) \rightarrow \ldots
$$

under the natural projections $\mathfrak{g}_{n}^{*} \rightarrow \mathfrak{g}_{n-1}^{*}$. Moreover, $\operatorname{Var}\left(J_{n-1}\right)=\overline{\operatorname{pr}_{n} \operatorname{Var}\left(J_{n}\right)}$ where the closure is taken in $\mathfrak{g}_{n-1}^{*}$.

Let $\mathfrak{k}_{1}$ be a simple finite-dimensional Lie algebra and $\mathfrak{k}_{2} \subset \mathfrak{k}_{1}$ be a simple finitedimensional subalgebra. The restriction of the Cartan-Killing of $\mathfrak{k}_{1}$ to $\mathfrak{k}_{2}$ is proportional to the Cartan-Killing form of $\mathfrak{k}_{2}$ with coefficient which we denote $\mathbb{I}_{\mathfrak{k}_{2}}^{\mathfrak{k}_{1}}$. This coefficient, known as the Dynkin index, is multiplicative: if $\mathfrak{k}_{3} \subset \mathfrak{k}_{2} \subset \mathfrak{k}_{1}$ is a chain of inclusions, then

$$
\mathfrak{I}_{\mathfrak{k}_{3}}^{\mathfrak{k}_{1}}=I_{\mathfrak{k}_{3}}^{\mathfrak{k}_{2}}{\mathfrak{\mathfrak { k } _ { 2 }}}_{\mathfrak{k}_{1}}^{\mathfrak{1}_{1}}
$$


The Dynkin index is always a positive integer Dy. Moreover, if $\mathfrak{k}_{2} \subset \mathfrak{k}_{1}$ are classical simple Lie algebras of the same type and of rank at least 5 , then $I_{\mathfrak{k}_{2}}^{\mathfrak{k}_{1}}=1$ if and only if the natural module of $\mathfrak{k}_{1}$ decomposes over $\mathfrak{k}_{2}$ as a natural plus a trivial module [DP, Proposition 2.3].

Proof of Theorem 3.1. Let $J$ be a non-zero Poisson ideal of locally infinite codimension in $\mathbf{S}\left(\mathfrak{g}_{\infty}\right)$. Without loss of generality we may assume that $J$ is a radical ideal, as the radical of a Poisson of locally infinite codimension ideal in $\mathbf{S}\left(\mathfrak{g}_{\infty}\right)$ is again Poisson and of locally infinite codimension.

Fix $n$ so that $J_{n}=J \cap \mathbf{S} \cdot\left(\mathfrak{g}_{n}\right)$ is non-zero and of infinite codimension in $\mathbf{S}\left(\mathfrak{g}_{n}\right)$. The image of any $G_{n+m}$-orbit in $\operatorname{Var}\left(J_{n+m}\right)$ under the morphism $\operatorname{Var}\left(J_{n+m}\right) \rightarrow \mathfrak{g}_{n}^{*}$ is not dense in $\mathfrak{g}_{n}^{*}$ as it lies in the proper closed subvariety $\operatorname{Var}\left(J_{n}\right) \subset \mathfrak{g}_{n}^{*}$. Therefore Proposition 4.2 implies that $\operatorname{dim}\left(\mathfrak{g}_{n} \cdot V_{n+m}\right)$ is bounded as a function on $m$. Hence the number of non-trivial simple $\mathfrak{g}_{n}$-constituents in $V_{n+m}$ and their dimensions are simultaneously bounded. As a consequence, $I_{\mathfrak{g}_{n}}^{\mathfrak{g}_{m+n}}$ is bounded as a function of $m>0$. Therefore there exists $N>0$ such that $I_{\mathfrak{g}_{n}}^{\mathfrak{g}_{n+1}}=1$ for all $n>N$. Now DP, Corollary 2.4] implies $\mathfrak{g}_{\infty} \cong \mathfrak{s l}_{\infty}, \mathfrak{s o}_{\infty}$ or $\mathfrak{s p}_{\infty}$.

Proof of Theorem 3.3. If $J$ is of locally finite codimension in $\mathbf{S}\left(\mathfrak{g}_{\infty}\right)$, then

$$
\operatorname{Var}(J)=0=\mathfrak{s} \leq 0 .
$$

Therefore, in the rest of the proof we can assume that $J$ is non-zero and of locally infinite codimension in $\mathbf{S}\left(\mathfrak{g}_{\infty}\right)$.

Fix $n$ so that $J_{n}=J \cap \mathbf{S}^{\prime}\left(\mathfrak{g}_{n}\right)$ is non-zero and of infinite codimension in $\mathbf{S}\left(\mathfrak{g}_{n}\right)$. Since for any non-zero $X \in \operatorname{Var}\left(J_{m+n}\right)$ the image in $\mathfrak{g}_{n}^{*}$ of the $G_{m+n}$-orbit $\mathcal{O}[X] \subset$ $\mathfrak{g}_{m+n}^{*}$ is not dense in $\mathfrak{g}_{n}^{*}$, Lemma 4.13 implies that $\operatorname{Var}\left(J_{m+n}\right) \subset \mathfrak{g}_{m+n}^{\leq r_{n}^{\prime}}$ for some $r_{n}^{\prime}$ which depends on $n$ only. Let $r_{n}$ be the minimal such $r_{n}^{\prime}$. By Lemma 4.13, $r_{n}<n$.

The inequality $r_{n}<n$ allows us to apply Lemma 4.14 when $m>n$. It implies that the image in $\mathfrak{g}_{n}^{\leq r_{n}}$ of $\mathcal{O}[X] \subset \mathfrak{g}_{m+n}^{*}$ is dense in $\mathfrak{g}_{n}^{\leq r_{n}}$ for any $X \in \operatorname{Var}\left(J_{m+n}\right)$ with $\operatorname{rk} X=r_{n}$. Furthermore, by definition, $r_{n} \leq r_{n+m}$. Lemma 4.7 implies that $r_{m+n} \geq r_{n}$, and therefore $r_{n}=r_{m+n}$. Set $r=r_{n}$. Then $J=J \leq r$.

\section{Some COROLlaries}

Corollary 3.2 implies that if $\mathfrak{g}_{\infty}$ is a locally simple Lie algebra which is not finitary, then any ideal in $U\left(\mathfrak{g}_{\infty}\right)$ is of locally finite codimension. Furthermore, a result of A. Zhilinskii [h2 claims that $\mathrm{U}\left(\mathfrak{g}_{\infty}\right)$ admits an ideal of locally finite codimension which is not the augmentation ideal if and only if $\mathfrak{g}_{\infty}$ is diagonal. These two statements yield the following corollary.

Corollary 6.1 (Baranov's Conjecture). Let $\mathfrak{g}_{\infty}$ be any locally simple Lie algebra. Then the augmentation ideal is the only non-zero ideal in $U\left(\mathfrak{g}_{\infty}\right)$ if and only if $\mathfrak{g}_{\infty}$ is not diagonal.

Furthermore, Theorems 3.1, 3.3 imply the following.

Corollary 6.2. Let $\mathfrak{g}_{\infty}$ be any locally simple Lie algebra and $I \subset \mathrm{U}\left(\mathfrak{g}_{\infty}\right)$ be an ideal. Then

a) $\operatorname{Var}(I) \neq 0$ implies that $\mathfrak{g}_{\infty} \cong \mathfrak{s l}_{\infty}, \mathfrak{s o} \mathfrak{o}_{\infty}, \mathfrak{s p} \mathfrak{p}_{\infty}$;

b) if $\mathfrak{g}_{\infty}=\mathfrak{s l}_{\infty}, \mathfrak{s o}_{\infty}, \mathfrak{s p}_{\infty}$ and $I \subset \mathrm{U}\left(\mathfrak{g}_{\infty}\right)$ is an ideal of locally infinite codimension, then $\operatorname{Var}(I)=\mathfrak{s}_{\infty}^{\leq r}$ for some $r \in \mathbb{Z}_{\geq 1}$. 
A. Zhilinskii [Zh3] (see also [h1]) has given a description of all ideals of locally finite codimension in $\mathrm{U}\left(\mathfrak{g}_{\infty}\right)$ for an arbitrary locally simple Lie algebra $\mathfrak{g}_{\infty}$. Therefore the problem of describing all ideals in $\mathrm{U}\left(\mathfrak{g}_{\infty}\right)$ gets reduced to the problem of describing all ideals of locally infinite codimension in $U\left(\mathfrak{g}_{\infty}\right)$ for $\mathfrak{g}_{\infty}=\mathfrak{s l}_{\infty}, \mathfrak{s o}_{\infty}, \mathfrak{s p}_{\infty}$. In the following two sections we will show in particular that all proj-varieties $\mathfrak{s} \leq r$ arise as associated "varieties" of ideals respectively of $\mathrm{U}\left(\mathfrak{s l}_{\infty}\right), \mathrm{U}\left(\mathfrak{s o}_{\infty}\right), \mathrm{U}\left(\mathfrak{s p}_{\infty}\right)$.

\section{Coherent local Systems of modules ANd a Classification of PRime INTEGRABLE IDEALS OF $\mathrm{U}\left(\mathfrak{g}_{\infty}\right)$}

In this section we review some published and unpublished results of A. Zhilinskii and draw corollaries.

Definition 7.1. An ideal $I \subset \mathrm{U}\left(\mathfrak{g}_{\infty}\right)$ is integrable if for any finitely generated subalgebra $U^{\prime} \subset \mathrm{U}\left(\mathfrak{g}_{\infty}\right)$, the ideal $I \cap U^{\prime}$ in $U^{\prime}$ is an intersection of ideals of finite codimension of $U^{\prime}$.

If a $\mathfrak{g}_{\infty}$-module $M$ is integrable, i.e. $\operatorname{dim} \mathrm{U}\left(\mathfrak{g}^{\prime}\right) m<\infty$ for any $m \in M$ and any finite-dimensional subalgebra $\mathfrak{g}^{\prime} \subset \mathfrak{g}_{\infty}$, the annihilator of $M$ in $\mathrm{U}\left(\mathfrak{g}_{\infty}\right)$ is an integrable ideal. Note that an equivalent definition of an integrable $\mathrm{U}\left(\mathfrak{g}_{\infty}\right)$ module is a left $\mathrm{U}\left(\mathfrak{g}_{\infty}\right)$-module $M$ for which $\operatorname{dim}\left(U^{\prime} m\right)<\infty$ for any $m \in M$ and any finitely generated subalgebra $U^{\prime} \subset \mathrm{U}\left(\mathfrak{g}_{\infty}\right)$. Integrable ideals in $\mathrm{U}\left(\mathfrak{g}_{\infty}\right)$ are described as annihilators of coherent local systems of finite-dimensional $\mathfrak{g}_{n}$-modules as introduced by A. Zhilinskii in Zh2. We discuss this topic below.

\subsection{Integrable ideals and coherent local systems.}

Definition 7.2. A coherent local system of modules (further shortened as c.l.s.) for $\mathfrak{g}_{\infty}=\lim _{\mathfrak{g}} \mathfrak{g}_{n}$ is a collection of sets

$$
\left\{Q_{n}\right\}_{n \in \mathbb{Z}_{\geq 1}} \subset \prod_{n \in \mathbb{Z}_{\geq 1}} \operatorname{Irr} \mathfrak{g}_{n}
$$

such that $Q_{m}=\left\langle Q_{n}\right\rangle_{m}$ for any $n>m$.

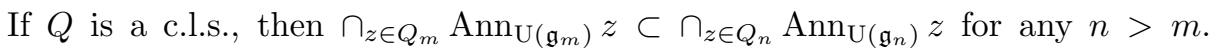
Therefore $\cup_{m}\left(\cap_{z \in Q_{m}} \operatorname{Ann}_{\mathrm{U}\left(\mathfrak{g}_{m}\right)} z\right)$ is an ideal of $\mathrm{U}(\mathfrak{g})$; we denote it by $I(Q)$. Note that $I(Q)$ is integrable.

A. Zhilinskii [Zh1, Zh2, Zh3 has classified c.l.s. for any locally simple Lie algebra $\mathfrak{g}_{\infty}$. Below we show how this classification leads to a description of integrable ideals of $\mathrm{U}\left(\mathfrak{g}_{\infty}\right)$ for $\mathfrak{g}_{\infty}=\mathfrak{s l}_{\infty}, \mathfrak{s o}_{\infty}, \mathfrak{s p}{ }_{\infty}$.

A c.l.s. $Q$ is irreducible if $Q \neq Q^{\prime} \cup Q^{\prime \prime}$ with $Q^{\prime} \notin Q^{\prime \prime}$ and $Q^{\prime \prime} \notin Q^{\prime}$. Let $Q$ be any c.l.s. and let $\mathcal{S}(Q)$ be the set of irreducible c.l.s. which are contained in $Q$. Then $\mathcal{S}(Q)$ has a finite subset of elements $Q(1), \ldots, Q(r)$ which are maximal by inclusion, and $Q=\cup_{r} Q(r)$ [Zh1] we call $Q(r)$ the irreducible components of $Q$. This makes apparent the analogy between c.l.s. and algebraic varieties.

Any integrable $\mathfrak{g}_{\infty}$-module $M$ determines a c.l.s. $Q:=\left\{Q_{n}\right\}_{n \in \mathbb{Z}_{\geq 0}}$, where

$$
Q_{n}:=\left\{z \in \operatorname{Irr} \mathfrak{g}_{n} \mid \operatorname{Hom}_{\mathfrak{g}_{n}}(z, M) \neq 0\right\}
$$

We denote this relation by $Q \leftarrow M$. We also recall that an integrable $\mathfrak{g}_{\infty}$-module $M$ is locally simple if $M=\lim _{\longrightarrow} M_{n}$ for a suitable chain $\ldots \subset M_{n} \subset M_{n+1} \subset \ldots$ of simple finite-dimensional $\mathfrak{g}_{n}$-submodules $M_{n}$ of $M$.

Proposition 7.3 ( [Zh1, Lemma 1.1.2]). If $Q$ is an irreducible c.l.s., then $I(Q)$ is the annihilator of some locally simple integrable $\mathfrak{g}_{\infty}$-module. In particular, $I(Q)$ is primitive and hence prime. 
Fix $n$. The set $\operatorname{Irrg}_{n}$ is parametrized by the lattice $\Lambda_{i}$ of integral dominant weights of $\mathfrak{g}_{n}$. Let $z_{1}, z_{2}$ be isomorphism classes of simple $\mathfrak{g}_{n}$-modules with respective highest weights $\lambda_{1}, \lambda_{2}$. We denote by $z_{1} z_{2}$ the isomorphism class of a simple module with highest weight $\lambda_{1}+\lambda_{2}$. If $S_{1}, S_{2} \subset \operatorname{Irr} \mathfrak{g}_{n}$ we set

$$
S_{1} S_{2}:=\left\{z \in \operatorname{Irrg} g_{n} \mid z=z_{1} z_{2} \text { for some } z_{1} \in S_{1} \text { and } z_{2} \in S_{2}\right\} \text {. }
$$

If $Q^{\prime}, Q^{\prime \prime}$ are c.l.s., we denote by $Q^{\prime} Q^{\prime \prime}$ the smallest c.l.s. such that $\left(Q^{\prime}\right)_{i}\left(Q^{\prime \prime}\right)_{i} \subset$ $\left(Q^{\prime} Q^{\prime \prime}\right)_{i}$. By definition, $Q^{\prime} Q^{\prime \prime}$ is the product of $Q^{\prime}$ and $Q^{\prime \prime}$. If $\mathfrak{g}_{\infty}=\mathfrak{s l}_{\infty}, \mathfrak{s p}_{\infty}$, then by [Zh1]

$$
\left(Q^{\prime}\right)_{n}\left(Q^{\prime \prime}\right)_{n}=\left(Q^{\prime} Q^{\prime \prime}\right)_{n} .
$$

7.2. Zhilinskii's classification of c.l.s. In this subsection we reproduce A. Zhilinskii's classification of irreducible c.l.s. for $\mathfrak{g}_{\infty} \cong \mathfrak{s l}_{\infty}, \mathfrak{s o}_{\infty}, \mathfrak{s p}_{\infty}$.

In the rest of the paper $\mathfrak{g}_{\infty}=\mathfrak{s l}_{\infty}, \mathfrak{s v _ { \infty }}$ or $\mathfrak{s p}_{\infty}$ and $\mathfrak{g}_{n}=\mathfrak{s}\left(V_{n}\right)$ is a sequence (1) of finite-dimensional simple Lie algebras satisfying the assumptions of Section 3 , We set $V_{\infty}:=\underline{\lim } V_{n}$ and $\left(V_{\infty}\right)_{*}:=\lim V_{n}^{*}$.

The following irreducible c.l.s. are by definition the basic c.l.s.:

$$
\begin{aligned}
& \text { for } \mathfrak{g}_{\infty}=\mathfrak{s l}_{\infty}: \mathcal{E} \leftarrow \Lambda^{\prime} V_{\infty}, \quad \mathcal{L}_{p} \leftarrow \Lambda^{p} V_{\infty}, \quad \mathcal{L}_{p}^{\infty} \leftarrow \mathbf{S}\left(V_{\infty} \otimes \mathbb{F}^{p}\right), \\
& \mathcal{R}_{q} \leftarrow \Lambda^{q}\left(V_{\infty}\right)_{*}, \quad \mathcal{R}_{q}^{\infty} \leftarrow \mathbf{S}\left(\left(V_{\infty}\right)_{*} \otimes \mathbb{F}^{q}\right), \quad \mathcal{E}^{\infty} \text { (all modules); } \\
& \text { for } \mathfrak{g}_{\infty}=\mathfrak{s p}_{\infty}: \mathcal{E} \leftarrow \Lambda^{\cdot} V_{\infty}, \quad \mathcal{L}_{p} \leftarrow \Lambda^{p} V_{\infty}, \quad \mathcal{L}_{p}^{\infty} \leftarrow \mathbf{S}\left(V_{\infty} \otimes \mathbb{F}^{p}\right), \\
& \mathcal{E}^{\infty} \text { (all modules); } \\
& \text { for } \mathfrak{g}_{\infty}=\mathfrak{s o}_{\infty}: \mathcal{E} \leftarrow \Lambda^{\cdot} V_{\infty}, \quad \mathcal{L}_{p} \leftarrow \Lambda^{p} V_{\infty}, \quad \mathcal{L}_{p}^{\infty} \leftarrow \mathbf{S}\left(V_{\infty} \otimes \mathbb{F}^{p}\right), \\
& \mathcal{R} \text { (spinor modules), } \mathcal{E}^{\infty} \text { (all modules), }
\end{aligned}
$$

where $p, q \in \mathbb{Z}_{\geq 1}$.

Proposition $\mathbf{7 . 4}$ (Unique factorization property [Zh1]). Any irreducible c.l.s. can be expressed uniquely as a product as follows:

$$
\begin{aligned}
& \left(\mathcal{L}_{v}^{\infty} \mathcal{L}_{v+1}^{x_{v+1}} \mathcal{L}_{v+2}^{x_{v+2}} \ldots \mathcal{L}_{n}^{x_{n}}\right) \mathcal{E}^{m}\left(\mathcal{R}_{w}^{\infty} \mathcal{R}_{w+1}^{z_{w+1}} \mathcal{R}_{w+2}^{z_{w+2}} \ldots \mathcal{R}_{n}^{z_{n}}\right) \quad \text { for } \mathfrak{g}_{\infty}=\mathfrak{s l}_{\infty}, \\
& \left(\mathcal{L}_{v}^{\infty} \mathcal{L}_{v+1}^{x_{v+1}} \mathcal{L}_{v+2}^{x_{v+2}} \ldots \mathcal{L}_{n}^{x_{n}}\right) \mathcal{E}^{m} \text { or }\left(\mathcal{L}_{v}^{\infty} \mathcal{L}_{v+1}^{x_{v+1}} \mathcal{L}_{v+2}^{x_{v+2}} \ldots \mathcal{L}_{n}^{x_{n}}\right) \mathcal{E}^{m} \mathcal{R} \text { for } \mathfrak{g}_{\infty}=\mathfrak{s o}_{\infty}, \\
& \left(\mathcal{L}_{v}^{\infty} \mathcal{L}_{v+1}^{x_{v+1}} \mathcal{L}_{v+2}^{x_{v+2}} \ldots \mathcal{L}_{n}^{x_{n}}\right) \mathcal{E}^{m} \quad \text { for } \mathfrak{g}_{\infty}=\mathfrak{s p}_{\infty},
\end{aligned}
$$

where

$$
\begin{gathered}
m, n, v, w \in \mathbb{Z}_{\geq 0}, v, w \leq n, \\
x_{i}, z_{j} \in \mathbb{Z}_{\geq 0} \text { for } v+1 \leq i \leq n \text { and } w+1 \leq j \leq n .
\end{gathered}
$$

Here, for $v=0, \mathcal{L}_{v}^{\infty}$ is assumed to be the identity (the c.l.s. consisting of the isomorphism class of the trivial 1-dimensional module at all levels), and for $w=0$, $\mathcal{R}_{w}^{\infty}$ is assumed to be the identity.

We say that an irreducible c.l.s. of $\mathfrak{s o}_{\infty}$ is of integer type if its expression does not contain $\mathcal{R}$, and of semiinteger type otherwise. Furthermore, we define a c.l.s. $Q$ to be of finite type if the set $Q_{n}$ is finite for all $n \geq 1$. It is easy to see that the ideal $I(Q)$ is of locally finite-codimension in $\mathrm{U}\left(\mathfrak{g}_{\infty}\right)$ if and only if $Q$ is of finite type.

If $\mathfrak{g}_{\infty}=\mathfrak{s l}_{\infty}, \mathfrak{s p}_{\infty}$, the irreducible c.l.s. of finite type form a free lattice (by means of product) generated by $\mathcal{E}, \mathcal{L}_{p}, \mathcal{R}_{q}$ for $\mathfrak{g}_{\infty}=\mathfrak{s l}_{\infty}$ and by $\mathcal{E}, \mathcal{L}_{p}$ for $\mathfrak{g}_{\infty}=\mathfrak{s p}_{\infty}$. For $\mathfrak{g}=\mathfrak{s o}_{\infty}$ the set of irreducible c.l.s. of finite type equals the union $\mathcal{N} \sqcup \mathcal{N} \mathcal{R}$, where $\mathcal{N}$ is a free lattice generated by $\mathcal{E}, \mathcal{L}_{p}$, and $\mathcal{N} \mathcal{R}:=\{N \mathcal{R} \mid N \in \mathcal{N}\}$ [Zh1].

7.3. Partial order by inclusion on c.l.s. To a c.l.s. $Q$ for $\mathfrak{s l}_{\infty}$ in the form (4) A. Zhilinskii assigns the following two non-increasing sequences of elements of $\mathbb{Z}_{\geq 0} \cup\{+\infty\}$ 


$$
\left\{l_{i}:=m+\Sigma_{j \geq i} x_{j}\right\}_{i} \text { and }\left\{r_{i}=m+\Sigma_{j \geq i} z_{j}\right\}_{i},
$$

where it is assumed $l_{1}=l_{2}=\ldots=l_{v}:=+\infty=: r_{1}=\ldots=r_{w}$. Note that

$$
\lim _{i \rightarrow \infty} l_{i}=\lim _{i \rightarrow \infty} r_{i}=m .
$$

Similarly, to a c.l.s. for $\mathfrak{s p}_{\infty}$ or $\mathfrak{s o}_{\infty}$ in the form (5) or (6) A. Zhilinskii assigns the non-increasing sequence

$$
\left\{l_{i}:=m+\Sigma_{j \geq i} x_{i}\right\}_{i},
$$

where $l_{1}=\ldots=l_{v}:=+\infty$. Again $\lim _{i \rightarrow \infty} l_{i}=m$.

Zhilinskii establishes the following inclusion criterion [Zh1. A c.l.s. $Q$ for $\mathfrak{s l}_{\infty}$ contains a c.l.s. $Q^{\prime}$ if and only if, for some $a, b \in \mathbb{Z}_{\geq 0}$, we have

$$
a+b=m-m^{\prime}, l_{i} \geq l_{i}^{\prime}+a, r_{i} \geq r_{i}^{\prime}+b
$$

for the corresponding sequences $\left\{l_{i}\right\},\left\{r_{i}\right\}$ and $\left\{l_{i}^{\prime}\right\},\left\{r_{i}^{\prime}\right\}$. If $Q$ and $Q^{\prime}$ are c.l.s. of $\mathfrak{s p}_{\infty}$, then $Q^{\prime} \subset Q$ if and only if

$$
l_{i} \geq l_{i}^{\prime} .
$$

Finally, if $Q$ and $Q^{\prime}$ are c.l.s. of $\mathfrak{s o}_{\infty}$, then $Q^{\prime} \subset Q$ if and only if $Q$ and $Q^{\prime}$ have the same integer or semiinteger type, and

$$
l_{i} \geq l_{i}^{\prime} .
$$

Corollary 7.5. If $\mathfrak{g}_{\infty}=\mathfrak{s l}_{\infty}, \mathfrak{s p}_{\infty}$, the only minimal c.l.s. is the trivial c.l.s. (i.e. the c.l.s. $Q$ with $Q_{n}$ being the isomorphism class of the 1-dimensional trivial $\mathfrak{g}_{n^{-}}$ module for all $n \geq 1$ ). If $\mathfrak{g}_{\infty}=\mathfrak{s o}_{\infty}$, there are two minimal c.l.s.: the trivial one and $\mathcal{R}$.

Proof. Follows from the inclusion criterion of A. Zhilinskii.

7.4. Tensor product and ideals. Fix $i$. If $S_{1}, S_{2} \subset \operatorname{Irr} \mathfrak{g}_{n}$ we set

$S_{1} \otimes S_{2}:=\left\{z \in \operatorname{Irr} \mathfrak{g}_{n} \mid \operatorname{Hom}\left(z, z_{1} \otimes z_{2}\right) \neq 0\right.$ for some $z_{1} \in S_{1}$ and $\left.z_{2} \in S_{2}\right\}$.

Furthermore, it is clear that the tensor product of two c.l.s. is a well defined c.l.s.. One can check that

$$
\begin{aligned}
& \left(\mathcal{L}_{v}^{\infty} \mathcal{L}_{v+1}^{x_{v+1}} \mathcal{L}_{v+2}^{x_{v+2}} \ldots \mathcal{L}_{n}^{x_{n}}\right) \mathcal{E}^{m}\left(\mathcal{R}_{w}^{\infty} \mathcal{R}_{w+1}^{z_{w+1}} \mathcal{R}_{w+2}^{z_{w+2}} \ldots \mathcal{R}_{n}^{z_{n}}\right)= \\
& \left(\mathcal{L}_{1}^{\infty}\right)^{\otimes v} \otimes\left(\mathcal{R}_{1}^{\infty}\right)^{\otimes w} \otimes\left(\left(\mathcal{L}_{1}^{x_{v+1}} \mathcal{L}_{2}^{x_{v+2}} \ldots \mathcal{L}_{n-v}^{x_{n}}\right) \mathcal{E}^{m}\left(\mathcal{R}_{1}^{z_{w+1}} \mathcal{R}_{2}^{z_{w+2}} \ldots \mathcal{R}_{n-w}^{z_{n}}\right)\right)
\end{aligned}
$$

for $\mathfrak{g}_{\infty}=\mathfrak{s l}_{\infty}$, and

$$
\begin{aligned}
& \left(\mathcal{L}_{v}^{\infty} \mathcal{L}_{v+1}^{x_{v+1}} \mathcal{L}_{v+2}^{x_{v+2}} \ldots \mathcal{L}_{n}^{x_{n}}\right) \mathcal{E}^{m}=\left(\mathcal{L}_{1}^{\infty}\right)^{\otimes v} \otimes\left(\left(\mathcal{L}_{1}^{x_{v+1}} \mathcal{L}_{2}^{x_{v+2}} \ldots \mathcal{L}_{n-v}^{x_{n}}\right) \mathcal{E}^{m}\right), \\
& \left(\mathcal{L}_{v}^{\infty} \mathcal{L}_{v+1}^{x_{v+1}} \mathcal{L}_{v+2}^{x_{v+2}} \ldots \mathcal{L}_{n}^{x_{n}}\right) \mathcal{E}^{m} \mathcal{R}=\left(\mathcal{L}_{1}^{\infty}\right)^{\otimes v} \otimes\left(\left(\mathcal{L}_{1}^{x_{v+1}} \mathcal{L}_{2}^{x_{v+2}} \ldots \mathcal{L}_{n-v}^{x_{n}}\right) \mathcal{E}^{m} \mathcal{R}\right)
\end{aligned}
$$

for $\mathfrak{g}_{\infty}=\mathfrak{s o}_{\infty}, \mathfrak{s p}_{\infty}$, where $\left(\mathcal{L}_{1}^{\infty}\right)^{\otimes v}:=\mathcal{L}_{1}^{\infty} \otimes \mathcal{L}_{1}^{\infty} \otimes \ldots \otimes \mathcal{L}_{1}^{\infty}(v$ times $)$. Note that formula (7) applies to $\mathfrak{s o}_{\infty}$-case only.

The above formulas yield a different parametrization of the irreducible c.l.s.: for $\mathfrak{g}_{\infty}=\mathfrak{s l}_{\infty}$ the irreducible c.l.s. are parametrized by triples $\left(v, w, Q_{f}\right)$, where $v, w \in \mathbb{Z}_{\geq 0}$ and $Q_{f}$ is an irreducible c.l.s. of finite type, and for $\mathfrak{g}_{\infty}=\mathfrak{s o}_{\infty}, \mathfrak{s p}_{\infty}$ the irreducible c.l.s. are parametrized by pairs $\left(v, Q_{f}\right)$, where $v \in \mathbb{Z}_{\geq 0}$ and $Q_{f}$ is an irreducible c.l.s. of finite type.

For $\mathfrak{g}_{\infty}=\mathfrak{s l}_{\infty}$ we set

$$
\operatorname{cls}\left(v, w, Q_{f}\right):=\left(\mathcal{L}_{1}^{\infty}\right)^{\otimes v} \otimes\left(\mathcal{R}_{1}^{\infty}\right)^{\otimes w} \otimes Q_{f}
$$

and

$$
I\left(v, w, Q_{f}\right):=I\left(\operatorname{cls}\left(v, w, Q_{f}\right)\right) .
$$


However, it is easy to check that the annihilators of $\mathcal{L}_{1}^{\infty}$ and $\mathcal{R}_{1}^{\infty}$ in $\mathrm{U}\left(\mathfrak{s l}_{\infty}\right)$ coincide. Therefore

$$
I\left(v, w, Q_{f}\right)=I\left(v+w, 0, Q_{f}\right) .
$$

In what follows we call an irreducible c.l.s. for $\mathfrak{g}_{\infty}=\mathfrak{s l}_{\infty}$ of the form $\operatorname{cls}\left(v, 0, Q_{f}\right)$ a left irreducible c.l.s. An arbitrary left c.l.s. is defined as a finite union of left irreducible c.l.s. We denote the left irreducible c.l.s. for $\mathfrak{g}_{\infty}=\mathfrak{s l}_{\infty}$ by $\operatorname{cls}\left(v, Q_{f}\right)$. We also set $I\left(v, Q_{f}\right):=I\left(v, 0, Q_{f}\right)$.

Note that for $\mathfrak{g}_{\infty}=\mathfrak{s o}_{\infty}, \mathfrak{s p}_{\infty}$ an arbitrary irreducible c.l.s. corresponds to a pair $\left(v, Q_{f}\right)$. Therefore the notations $\operatorname{cls}\left(v, Q_{f}\right)$ and $I\left(v, Q_{f}\right)$ make sense in all three cases: $\mathfrak{s l}_{\infty}, \mathfrak{s o}_{\infty}, \mathfrak{s p}_{\infty}$.

7.5. Classification of integrable ideals in $\mathrm{U}\left(\mathfrak{g}_{\infty}\right)$. Recall that $\mathfrak{g}_{n}=\mathfrak{s l}_{n+1}$ for $\mathfrak{g}_{\infty} \cong \mathfrak{s l}_{\infty}$. In this case the space of weights of $\mathfrak{g}_{n}$ is identified with the set of $(n+1)$-tuples $\left(\lambda_{1}, \ldots, \lambda_{n+1}\right), \lambda_{i} \in \mathbb{F}$, up to the equivalence relation

$$
\left(\lambda_{1}, \ldots, \lambda_{n+1}\right) \sim\left(\lambda_{1}+k, \ldots, \lambda_{n+1}+k\right)
$$

for $k \in \mathbb{F}$. The lattice of integral dominant weights is identified with the set of weights such that $\lambda_{i+1}-\lambda_{i} \in \mathbb{Z}_{\geq 0}$ for $1 \leq i \leq n-1$.

For $\mathfrak{g}_{\infty} \cong \mathfrak{s p}_{\infty}$, the space of weights of $\mathfrak{g}_{n} \cong \mathfrak{s p}_{2 n}$ is identified with the set of $n$-tuples $\left(\lambda_{1}, \ldots, \lambda_{n}\right), \lambda_{i} \in \mathbb{F}$. The lattice of integral dominant weights is identified with the set of weights such that $\lambda_{i} \in \mathbb{Z}_{\geq 0}$ and $\lambda_{i+1} \geq \lambda_{i}$ for $1 \leq i \leq n-1$.

For $\mathfrak{g}_{\infty} \cong \mathfrak{s o}_{\infty}$, we assume that $\mathfrak{g}_{n} \cong \mathfrak{s o}_{2 n}$. The space weights of $\mathfrak{g}_{n}=\mathfrak{s o}_{2 n}$ is identified with the set of $n$-tuples $\left(\lambda_{1}, \ldots, \lambda_{n}\right), \lambda_{i} \in \mathbb{F}$. The lattice of integral dominant weights is identified with the set of weights such that $\lambda_{i} \in \frac{1}{2} \mathbb{Z}, \lambda_{i+1}-\lambda_{i} \in$ $\mathbb{Z}_{\leq 0}$ for $1 \leq i \leq n-1$ and $\lambda_{n-1} \geq\left|\lambda_{n}\right|$.

Let $\mathcal{W}_{n}$ denote the Weyl group of $\mathfrak{g}_{n}$, and let $\rho_{n}$ be the half-sum of positive roots of $\mathfrak{g}_{n}$. The set of radical ideals of $Z_{\mathrm{U}\left(\mathfrak{g}_{n}\right)}$ is identified with the set of Zariski-closed $\mathcal{W}_{n}$-stable subsets of the space of weights of $\mathfrak{g}_{n}$.

Let $Q$ be a c.l.s. For any $n \in \mathbb{Z}_{\geq 1}$ we denote by $\overline{Q_{n}}$ the Zariski-closure of the set of highest weights of the isomorphism classes of simple $\mathfrak{g}_{n}$-modules from $Q_{n}$ in the space of weights of $\mathfrak{g}_{n}$.

Lemma 7.6. a) For any $v \in \mathbb{Z}_{\geq 0}$ and any c.l.s. $Q_{f}$ of finite type, the following conditions are equivalent:

(i) $\left(v, Q_{f}\right)=\left(v^{\prime}, Q_{f}^{\prime}\right)$,

(ii) $\mathcal{W}_{n}\left(\rho_{n}+\overline{\operatorname{cls}\left(v, Q_{f}\right)_{n}}\right)=\mathcal{W}_{n}\left(\rho_{n}+\overline{\left.\operatorname{cls}\left(v^{\prime}, Q_{f}^{\prime}\right)_{n}\right)}\right.$ for all $n \in \mathbb{Z}_{\geq 1}$.

b) Let $z \in \operatorname{Irr} \mathfrak{g}_{n}$ and let $\lambda_{z}$ be the highest weight of a representative of $z$. For $\mathfrak{g}_{\infty} \cong \mathfrak{s o}_{\infty}, \mathfrak{s p}_{\infty}$, we have

$$
\lambda_{z}+\rho_{n} \in \mathcal{W}_{n}\left(\rho_{n}+\overline{\operatorname{cls}\left(v, Q_{f}\right)_{n}}\right)
$$

if and only if $z \in \operatorname{cls}\left(v, Q_{f}\right)_{n}$. For $\mathfrak{g}_{\infty} \cong \mathfrak{s l}_{\infty}$, we have

$$
\lambda_{z}+\rho_{n} \in \mathcal{W}_{n}\left(\rho_{n}+\overline{\operatorname{cls}\left(v, Q_{f}\right)_{n}}\right)
$$

if and only if there exist $v^{\prime}, v^{\prime \prime} \in \mathbb{Z}_{\geq 0}$ such that $v^{\prime}+v^{\prime \prime}=v$ and $z \in \operatorname{cls}\left(v^{\prime}, v^{\prime \prime}, Q_{f}\right)_{n}$. c) Let $v \in \mathbb{Z}_{\geq 0}$ and $Q_{f}$ be a c.l.s. for $\mathfrak{g}_{\infty}$. Then, for $\mathfrak{g}_{\infty} \cong \mathfrak{s o}_{\infty}, \mathfrak{s p}_{\infty}$ we have $Q\left(I\left(v, Q_{f}\right)\right)=\operatorname{cls}\left(v, Q_{f}\right)$. For $\mathfrak{g}_{\infty} \cong \mathfrak{s l}_{\infty}$ we have

$$
Q\left(I\left(v, Q_{f}\right)\right)=\cup_{v^{\prime}+v^{\prime \prime}=v} \operatorname{cls}\left(v^{\prime}, v^{\prime \prime}, Q_{f}\right) .
$$

Proof. Part a) follows from the following explicit description of the $\operatorname{closure} \overline{\operatorname{cls}\left(v, Q_{f}\right)_{n+v}}$ : if $Q_{f}$ is an irreducible c.l.s. of finite type, then $\left(\lambda_{1}, \ldots, \lambda_{n+v}\right) \in \overline{\operatorname{cls}\left(v, Q_{f}\right)_{n+v}}$ if and only if the simple $\mathfrak{g}_{n}$-module with highest weight $\left(\lambda_{v+1}, \ldots, \lambda_{n+v}\right)$ lies in $\left(Q_{f}\right)_{n}$. 
Part b) is a straightforward corollary of part a). We proceed to c).

We fix $v, Q_{f}, n$. Let $z \in Q\left(I\left(v, Q_{f}\right)\right)_{n}$ and $\lambda_{z}$ be the highest weight of $z$. By definition, $I \cap \mathrm{U}\left(\mathfrak{g}_{n}\right) \subset \operatorname{Ann}_{\mathrm{U}\left(\mathfrak{g}_{n}\right)} z$. Then $I \cap \mathrm{Z}\left(\mathrm{U}\left(\mathfrak{g}_{n}\right)\right) \subset\left(\operatorname{Ann}_{\mathrm{U}\left(\mathfrak{g}_{n}\right)} z\right) \cap \mathrm{Z}\left(\mathrm{U}\left(\mathfrak{g}_{n}\right)\right)$. This inclusion is equivalent to the condition

$$
\lambda_{z}+\rho_{n} \in \mathcal{W}_{n}\left(\rho_{n}+\overline{\operatorname{cls}\left(v, Q_{f}\right)_{n}}\right) .
$$

Therefore $z \in \operatorname{cls}\left(v, Q_{f}\right)_{n}$ for $\mathfrak{g}_{\infty} \cong \mathfrak{s p}_{\infty}, \mathfrak{s o}_{\infty}$, and $z \in \cup_{v^{\prime}+v^{\prime \prime}=v} \operatorname{cls}\left(v^{\prime}, v^{\prime \prime}, Q_{f}\right)_{n}$ for $\mathfrak{g}_{\infty} \cong \mathfrak{s l}_{\infty}$ by b).

Hence $Q\left(I\left(v, Q_{f}\right)\right)=\operatorname{cls}\left(v, Q_{f}\right)$ for $\mathfrak{g}_{\infty} \cong \mathfrak{s o}_{\infty}, \mathfrak{s p}_{\infty}$.

Assume that $\mathfrak{g}_{\infty} \cong \mathfrak{s l}_{\infty}$. Then $I\left(v^{\prime}, v^{\prime \prime}, Q_{f}\right)=I\left(v^{\prime}+v^{\prime \prime}, Q_{f}\right)$ and thus $\operatorname{cls}\left(v^{\prime}, v^{\prime \prime}, Q_{f}\right) \subset$ $Q\left(I\left(v^{\prime}+v^{\prime \prime}, Q_{f}\right)\right)$. Hence $Q\left(I\left(v, Q_{f}\right)\right) \cup_{v^{\prime}+v^{\prime \prime}=v} \operatorname{cls}\left(v^{\prime}, v^{\prime \prime}, Q_{f}\right)$.

For any ideal $I \subset \mathrm{U}\left(\mathfrak{g}_{\infty}\right)$, set

$$
Q(I)_{n}:=\left\{z \in \operatorname{Irr} \mathfrak{g}_{n} \mid I \cap \mathrm{U}\left(\mathfrak{g}_{n}\right) \subset \operatorname{Ann}_{\mathrm{U}\left(\mathfrak{g}_{n}\right)} z\right\}
$$

and note that $Q(I)$ is a well-defined c.l.s.. Note that if $I$ is integrable, then $I$ is the annihilator of $Q(I)$.

Proposition 7.7. Assume $\mathfrak{g}_{\infty}=\mathfrak{s l}_{\infty}, \mathfrak{s o}_{\infty}, \mathfrak{s p}_{\infty}$. An integrable ideal of $\mathrm{U}\left(\mathfrak{g}_{\infty}\right)$ is prime if and only if it is primitive. 2

Proof. Let $I$ be a prime integrable ideal of $\mathrm{U}\left(\mathfrak{g}_{\infty}\right)$. Then $I$ is the annihilator of $Q(I)$. Let $Q_{1}, \ldots, Q_{s}$ be the irreducible components of $Q(I)$. We have

$$
I=I(Q(I)) \subset \cap_{i \leq s} I\left(Q_{s}\right)
$$

and

$$
I\left(Q_{1}\right) I\left(Q_{2}\right) \ldots I\left(Q_{s}\right) \subset I(Q(I))=I .
$$

Therefore $I=I(Q(I))$ coincides with $I\left(Q_{i}\right)$ for some irreducible c.l.s. $Q_{i}$. Thus the ideal $I\left(Q_{i}\right)$ is primitive by Proposition 7.3 . On the other hand, any primitive ideal is prime.

For $\mathfrak{g}_{\infty}=\mathfrak{s l}_{\infty}$, we denote by $Q_{l}(I)$ the union of all left irreducible components of $Q(I)$. For $\mathfrak{g}_{\infty}=\mathfrak{s o}_{\infty}, \mathfrak{s p}_{\infty}$, we put $Q_{l}(I)=Q(I)$.

Theorem 7.8. a) The maps

$$
\begin{gathered}
I \mapsto Q_{l}(I), \\
Q \mapsto I(Q)
\end{gathered}
$$

are mutually inverse bijections between the set of prime integrable ideals $I \subset \mathrm{U}\left(\mathfrak{g}_{\infty}\right)$ and the set of irreducible left c.l.s..

b) If $\mathfrak{g}_{\infty}=\mathfrak{s o}_{\infty}, \mathfrak{s p}_{\infty}$, the maps in a) extend to mutually inverse anti-isomorphisms

$$
\begin{aligned}
& I \mapsto Q(I), \\
& Q \mapsto I(Q)
\end{aligned}
$$

between the lattice of integrable ideals in $\mathrm{U}\left(\mathfrak{g}_{\infty}\right)$ and the lattice of c.l.s. for $\mathfrak{g}_{\infty} 3$. c) If $\mathfrak{g}_{\infty}=\mathfrak{s l}_{\infty}$, any integrable ideal of $\mathrm{U}\left(\mathfrak{s l}_{\infty}\right)$ equals $I(Q)$ for some left c.l.s. $Q$ for $\mathfrak{g}_{\infty}$.

\footnotetext{
${ }^{2}$ The analogous statement is false for $\mathfrak{g}_{n}$. For instance, $I\left(\mathcal{L}_{1}\right) \cap \mathrm{U}\left(\mathfrak{g}_{n}\right), n \geq 2$, is an integrable prime ideal of $\mathrm{U}\left(\mathfrak{g}_{n}\right)$ which is not primitive.

${ }^{3}$ Contrary to our conventions from Section 2 here we consider $\mathrm{U}\left(\mathfrak{g}_{\infty}\right)$ as an integrable ideal and $\{0\}$ as c.l.s.
} 
Proof. Let $I$ be a prime integrable ideal of $\mathrm{U}\left(\mathfrak{g}_{\infty}\right)$. By definition, $I$ is the annihilator of $Q(I)$. Let $Q_{1}, \ldots, Q_{s}$ be the irreducible components of $Q(I)$. Then

$$
I=I(Q(I)) \subset \cap_{i \leq s} I\left(Q_{s}\right)
$$

and

$$
I\left(Q_{1}\right) I\left(Q_{2}\right) \ldots I\left(Q_{s}\right) \subset I(Q(I))=I .
$$

Therefore $I=I(Q(I))$ coincides with $I\left(Q_{i}\right)$ for some irreducible c.l.s. $Q_{i}$. Hence, by Subsection 7.4 $I=I\left(v, Q_{f}\right)$ for some $v \in \mathbb{Z}_{\geq 0}$ and some c.l.s. of finite type $Q_{f}$.

To prove a) it remains to check that the annihilators of the c.l.s. corresponding to distinct pairs $\left(v, Q_{f}\right)$ and $\left(v^{\prime}, Q_{f}^{\prime}\right)$ are distinct. For $\mathfrak{g}_{\infty}=\mathfrak{s l}_{\infty}$ we have to prove that $I\left(v, 0, Q_{f}\right)=I\left(v^{\prime}, 0, Q_{f}^{\prime}\right)$ if and only if $v=v^{\prime}$ and $Q_{f}=Q_{f}^{\prime}$. For $\mathfrak{g}_{\infty}=\mathfrak{s o}_{\infty}, \mathfrak{s p}_{\infty}$, we have to check that $I\left(v, Q_{f}\right)=I\left(v^{\prime}, Q_{f}^{\prime}\right)$ if and only if $v=v^{\prime}$ and $Q_{f}=Q_{f}^{\prime}$. Both statements follow from Lemma 7.6. Hence, a) is proved.

To prove b) we assume that $\mathfrak{g}_{\infty} \cong \mathfrak{s o}_{\infty}, \mathfrak{s p}_{\infty}$. Let $Q$ be any c.l.s.. Then $Q=$ $\cup_{i \leq s} Q_{i}$ for some irreducible c.l.s. $Q_{i}$, and $I(Q)=\cap_{i \leq s} I\left(Q_{i}\right)$. On the other hand, $Q_{l}\left(\cap_{i \leq s} I\left(Q_{i}\right)\right)=Q\left(\cap_{i \leq s} I\left(Q_{i}\right)\right)$ by definition, and

$$
Q\left(\cap_{i \leq s} I\left(Q_{i}\right)\right)=\cup_{i \leq s}\left(Q\left(I\left(Q_{i}\right)\right)\right)=\cup_{i \leq s} Q_{i}=Q
$$

by a). Therefore the maps (8) and (9) are mutually inverse. In addition, it is now obvious that both maps are anti-homomorphisms of lattices. This proves b).

To prove c) we assume that $\mathfrak{g}_{\infty} \cong \mathfrak{s l}_{\infty}$. Let $I$ be an integrable ideal of $\mathrm{U}\left(\mathfrak{s l}_{\infty}\right)$. Then $I=\cap_{i \leq s} I_{s}$, where $I_{i}$ are prime integrable ideals of $\mathrm{U}\left(\mathfrak{s l}_{\infty}\right)$. For any $i \leq s$, $I_{i}=I\left(v_{i},\left(Q_{f}\right)_{i}\right)$ for some $\left(v_{i},\left(Q_{f}\right)_{i}\right)$ as in a). In particular, $I_{i}$ is the annihilator of a left local system $Q_{i}:=\left(v_{i}, 0,\left(Q_{f}\right)_{i}\right)$. Then $I$ is the annihilator of $\cup_{i \leq s} Q_{i}$. This proves c).

Note that for $\mathfrak{g}_{\infty} \cong \mathfrak{s l}_{\infty}$ the annihilators of $\mathcal{L}_{2}^{\infty}$ and $\left(\mathcal{L}_{2}^{\infty}\right) \cup\left(\mathcal{L}_{1}^{\infty} \mathcal{R}_{1}\right)$ coincide. This shows in particular that the one-to-one correspondence between left irreducible c.l.s. of $\mathfrak{g}_{\infty}$ and prime integrable ideals of $\mathrm{U}\left(\mathfrak{g}_{\infty}\right)$ can not be extended to an antiisomorphism between the corresponding lattices.

Nevertheless, Theorem $7.8 \mathrm{c}$ ) provides a certain description of general integrable ideal of $U\left(\mathfrak{s l}_{\infty}\right)$ : it yields a surjection from the set of c.l.s. of $\mathfrak{s l}_{\infty}$ to the set of integrable ideals of $\mathrm{U}\left(\mathfrak{s l}_{\infty}\right)$. Two c.l.s. $Q_{1}, Q_{2}$ determine the same integrable ideal $I\left(Q_{1}\right)=I\left(Q_{2}\right)$ if and only if $Q\left(I\left(Q_{1}\right)\right)=Q\left(I\left(Q_{2}\right)\right)$. For any irreducible c.l.s. $Q$, the c.l.s. $Q(I(Q))$ is described by Lemma 7.6 c). If $Q$ is a reducible c.l.s., i.e. $Q=\cup_{j \leq s} Q_{j}$ for some irreducible c.l.s. $Q_{1}, \ldots, Q_{j}$, then $Q(I(Q))=\cup_{j \leq s} Q\left(I\left(Q_{j}\right)\right)$. This allows in principle to check when $Q\left(I\left(Q_{1}\right)\right)=Q\left(I\left(Q_{2}\right)\right)$.

Furthermore, let's point out that Theorem 7.8, together with A. Zhilinskii's result [Zh1], Zh3 that any coherent local system is the union of finitely many coherent local systems, implies that the lattice of integrable ideals of $U\left(\mathfrak{g}_{\infty}\right)$ satisfies the ascending chain condition. This has already been stated in [Zh1 and Zh3]. We would like to think of this result as of "relative Nötherianity" of the algebra $\mathrm{U}\left(\mathfrak{g}_{\infty}\right)$. We don't know whether $\mathrm{U}\left(\mathfrak{g}_{\infty}\right)$ is two-sided Nötherian.

Theorem 7.8 and Corollary 7.5 imply immediately that if $\mathfrak{g}_{\infty}=\mathfrak{s l}_{\infty}, \mathfrak{s p}_{\infty}$, the augmentation ideal is the unique integrable maximal ideal of $\mathrm{U}\left(\mathfrak{g}_{\infty}\right)$. For $\mathfrak{g}_{\infty}=\mathfrak{s o}_{\infty}$ there are two integrable maximal ideals: the augmentation ideal and the "spinor ideal" $I(\mathcal{R})$. 
Corollary 7.9. The algebras $\mathrm{U}\left(\mathfrak{s l}_{\infty}\right), \mathrm{U}\left(\mathfrak{s o}_{\infty}\right)$ and $\mathrm{U}\left(\mathfrak{s p}_{\infty}\right)$ are pairwise nonisomorphic.

Proof. The algebras $\mathrm{U}\left(\mathfrak{s l}_{\infty}\right), \mathrm{U}\left(\mathfrak{s p}_{\infty}\right)$ have each a unique integrable maximal ideal, while the algebra $\mathrm{U}\left(\mathfrak{s o}_{\infty}\right)$ has two integrable maximal ideals. Hence $\mathrm{U}\left(\mathfrak{s l}_{\infty}\right) \neq$ $\mathrm{U}\left(\mathfrak{s o}_{\infty}\right)$ and $\mathrm{U}\left(\mathfrak{s p}_{\infty}\right) \not \mathrm{U}\left(\mathfrak{s o}_{\infty}\right)$.

Consider now prime submaximal integrable ideals in $U\left(\mathfrak{s l}_{\infty}\right)$ and $U\left(\mathfrak{s p}_{\infty}\right)$, i.e. integrable prime ideals which are properly contained only in integrable maximal ideals. Using the inclusion criterion of irreducible c.l.s. from Subsection [7.3, one checks immediately (using Theorem $7.8 \mathrm{a})$ ) that $\mathrm{U}\left(\mathfrak{s l}_{\infty}\right)$ has two such submaximal ideals, namely $I\left(\mathcal{L}_{1}\right)$ and $I\left(\mathcal{R}_{1}\right)$, while $\mathrm{U}\left(\mathfrak{s p}_{\infty}\right)$ has a single such ideal $I\left(\mathcal{L}_{1}\right)$. Hence $\mathrm{U}\left(\mathfrak{s l}_{\infty}\right) \neq \mathrm{U}\left(\mathfrak{s p}_{\infty}\right)$.

Finally, note that

$$
\operatorname{Var}\left(I\left(v, Q_{f}\right)\right)= \begin{cases}\mathfrak{s l} \mathfrak{l l}_{\infty}^{\leq v} & \text { for } \mathfrak{g}_{\infty}=\mathfrak{s l} \mathfrak{l}_{\infty}, \\ \mathfrak{s o}{ }_{\infty}^{\leq 2 v} & \text { for } \mathfrak{g}_{\infty}=\mathfrak{s o}_{\infty}, \\ \mathfrak{s p} \mathfrak{\infty}_{\infty}^{\leq 2 v} & \text { for } \mathfrak{g}_{\infty}=\mathfrak{s p}_{\infty} .\end{cases}
$$

Since $\mathfrak{s o} \leq_{\infty}^{\leq 2 v+1}=\mathfrak{s o} \leq_{\infty}^{\leq 2 v}$, all possible associated "varieties" of ideals in $\mathrm{U}\left(\mathfrak{s l}_{\infty}\right)$ and $\mathrm{U}\left(\mathfrak{s o}_{\infty}\right)$ appear in the right-hand side of (10). The only possible associated "varieties" which do not appear in the right-hand side of (10) are $\mathfrak{s p}_{\infty}^{\leq 2 v+1}$ for $v \in \mathbb{Z}_{\geq 0}$, and these proj-varieties are not associated "varieties" of integrable ideals of $\mathrm{U}\left(\mathfrak{s p}_{\infty}\right)$.

\section{Some NON-INTEGRABLE IDEALS OF $\mathrm{U}\left(\mathfrak{s p}_{\infty}\right)$}

We will now provide non-integrable ideals of $\mathrm{U}\left(\mathfrak{s p}_{\infty}\right)$ whose associated "varieties" are the proj-varieties $\mathfrak{s p}_{\infty}^{\leq 2 v+1}$ for $z \in \mathbb{Z}_{\geq 0}$. We start with a lemma and a corollary.

Let $\mathfrak{g}$ be a semisimple Lie algebra. Consider the coproduct

$$
\Delta: \mathrm{U}(\mathfrak{g}) \rightarrow \mathrm{U}(\mathfrak{g}) \otimes \mathrm{U}(\mathfrak{g})(x \mapsto x \otimes 1+1 \otimes x \text { for } x \in \mathfrak{g}) .
$$

In what follows we will denote the "diagonal", "left" and "right" copies of $\mathfrak{g}$ respectively by $\mathfrak{g}_{\Delta}, \mathfrak{g}_{l}, \mathfrak{g}_{r}$, i.e. we have

$$
\Delta: \mathrm{U}\left(\mathfrak{g}_{\Delta}\right) \subset \mathrm{U}\left(\mathfrak{g}_{l}\right) \otimes \mathrm{U}\left(\mathfrak{g}_{r}\right) .
$$

Lemma 8.1. Let $I_{l}, I_{r}$ be ideals of $\mathrm{U}(\mathfrak{g})$. Then

$$
\operatorname{Var}\left(I_{l}\right)+\operatorname{Var}\left(I_{r}\right) \subset \operatorname{Var}\left(\mathrm{U}\left(\mathfrak{g}_{\Delta}\right) /\left(\mathrm{U}\left(\mathfrak{g}_{\Delta}\right) \cap\left(I_{l} \otimes 1+1 \otimes I_{r}\right)\right)\right),
$$

where $\operatorname{Var}\left(I_{l}\right)+\operatorname{Var}\left(I_{r}\right)$ is a pointwise sum of the varieties $\operatorname{Var}\left(I_{l}\right)$ and $\operatorname{Var}\left(I_{r}\right)$ inside $\mathfrak{g}^{*}$.

Proof. We have

$\operatorname{gr}\left(\mathrm{U}\left(\mathfrak{g}_{\Delta}\right) \cap\left(I_{l} \otimes \mathrm{U}\left(\mathfrak{g}_{r}\right)+\mathrm{U}\left(\mathfrak{g}_{l}\right) \otimes I_{r}\right)\right) \subset \operatorname{gr}\left(I_{l} \otimes \mathrm{U}\left(\mathfrak{g}_{r}\right)+\mathrm{U}\left(\mathfrak{g}_{l}\right) \otimes I_{r}\right)=\operatorname{gr} I_{l} \otimes \mathrm{S}^{\cdot}\left(\mathfrak{g}_{r}\right)+\mathrm{S} \cdot\left(\mathfrak{g}_{l}\right) \otimes \operatorname{gr} I_{r}$.

Therefore

$$
\operatorname{Var}\left(\operatorname{gr}\left(I_{l}\right)\right)+\operatorname{Var}\left(\operatorname{gr}\left(I_{r}\right)\right) \subset \operatorname{Var}\left(\operatorname{gr}\left(\mathrm{U}\left(\mathfrak{g}_{\Delta}\right) \cap\left(I_{l} \otimes \mathrm{U}\left(\mathfrak{g}_{r}\right)+\mathrm{U}\left(\mathfrak{g}_{l}\right) \otimes I_{r}\right)\right)\right) .
$$

Corollary 8.2. Let $M_{l}, M_{r}$ be $\mathfrak{g}$-modules with annihilators $I_{l}, I_{r}$. Let $I_{\Delta}$ be the annihilator of $M_{l} \otimes M_{r}$. Then

$$
\operatorname{Var}\left(I_{l}\right)+\operatorname{Var}\left(I_{r}\right) \subset \operatorname{Var}\left(I_{\Delta}\right) \text {. }
$$


Proof. We first consider $M_{l} \otimes M_{r}$ as $\mathrm{U}\left(\mathfrak{g}_{l}\right) \otimes \mathrm{U}\left(\mathfrak{g}_{r}\right)$-module. Then the structure of $\mathfrak{g}$-module on $M_{l} \otimes M_{r}$ comes from the homomorphism $\Delta$. Therefore

$$
\operatorname{Ann}_{\mathrm{U}(\mathfrak{g})}\left(M_{1} \otimes M_{2}\right)=\mathrm{U}\left(\mathfrak{g}_{\Delta}\right) \cap\left(I_{l} \otimes 1+1 \otimes I_{r}\right) .
$$

Hence $\operatorname{Var}\left(I_{l}\right)+\operatorname{Var}\left(I_{r}\right) \subset \operatorname{Var}\left(I_{\Delta}\right)$ by Lemma 8.1

We now provide a maximal ideal $I \subset \mathrm{U}\left(\mathfrak{s p}_{\infty}\right)$ such that $\operatorname{Var}(I)=\mathfrak{s p}(V) \leq 1$.

Consider the Weyl algebra $\mathbb{W}_{\infty}$ of infinitely many variables, i.e. the associative algebra generated by $x_{1}, x_{2}, \ldots, x_{n}, \ldots, \partial_{x_{1}}, \partial_{x_{2}}, \ldots, \partial_{x_{n}}, \ldots$ with relations

$$
\begin{gathered}
{\left[x_{i}, x_{j}\right]=0, \quad\left[\partial_{x_{i}}, \partial_{x_{j}}\right]=0 \text { for all } i, j ;} \\
{\left[\partial_{x_{i}}, x_{i}\right]=1 \text { for } 1 \leq i \leq n-1 ; \quad\left[\partial_{x_{i}}, x_{j}\right]=0 \text { for all } i \neq j .}
\end{gathered}
$$

The subspace spanned by $1, x_{i} x_{j}, x_{i} \partial_{x_{j}}, \partial_{x_{j}} \partial_{x_{i}}$ is a Lie subalgebra of the Lie algebra associated with $\mathbb{W}_{\infty}$. This subalgebra $\tilde{\mathfrak{s}}$ has a 1-dimensional center generated by 1 , and its derived subalgebra $[\tilde{\mathfrak{s}}, \tilde{\mathfrak{s}}]$ is isomorphic to $\mathfrak{s p}_{\infty}$. Therefore we have a homomorphism $\mathrm{U}\left(\mathfrak{s p}_{\infty}\right) \rightarrow \mathbb{W}_{\infty}$; the kernel of this homomorphism is an ideal $I_{\mathbb{W}}$. For any $n, \mathfrak{g}_{n}=\mathfrak{s p}_{2 n}$ and $I_{\mathbb{W}} \cap \mathrm{U}\left(\mathfrak{g}_{n}\right)$ is a Joseph ideal [Jo. We denote by $V_{\mathbb{W}}$ the algebra $\mathbb{F}\left[x_{1}, x_{2}, \ldots\right]$ considered as an $\mathfrak{s p}_{\infty}$-module.

It is well known that the ideal $I_{\mathbb{W}} \cap \mathrm{U}\left(\mathfrak{g}_{n}\right)$ is maximal (thus primitive and prime) in $\mathrm{U}\left(\mathfrak{s p}_{2 n}\right)$ and not integrable. Hence $I_{\mathbb{W}}$ is a maximal ideal in $\mathrm{U}\left(\mathfrak{s p}_{\infty}\right)$ which is not integrable. The zero-set $\operatorname{Var}\left(I_{\mathbb{W}}\right)$ coincides with $\mathfrak{s p} \leq 1$ (note that any element of rank 1 in $\mathfrak{s p}_{2 n}$ is nilpotent).

More generally, we have the following.

Proposition 8.3. For any $v \in \mathbb{Z}_{\geq 0}$ we have

$$
\operatorname{Var}\left(\operatorname{Ann}_{\mathrm{U}\left(\mathfrak{s p}_{\infty}\right)}\left(\mathbf{S} \cdot\left(V_{\infty} \otimes \mathbb{F}^{v}\right) \otimes V_{\mathbb{W}}\right)\right)=\mathfrak{s p}_{\infty}^{\leq 2 v+1} .
$$

Proof. By Corollary 8.2, we have

$$
\begin{gathered}
\overline{\operatorname{Var}\left(\operatorname{Ann}_{\mathrm{U}\left(\mathfrak{s p}_{\infty}\right)}\left(\mathbf{S}^{\cdot}\left(V_{\infty} \otimes \mathbb{F}^{v}\right)\right)\right)+\operatorname{Var}\left(I_{\mathbb{W}}\right)}=\overline{\mathfrak{s p}_{\infty}^{\leq 2 v}+\mathfrak{s p}_{\infty}^{\leq 1}}= \\
=\mathfrak{s p}_{\infty}^{\leq 2 v+1} \subset \operatorname{Var}\left(\operatorname{Ann}_{\mathrm{U}\left(\mathfrak{s p}_{\infty}\right)}\left(\mathbf{S}\left(V_{\infty} \otimes \mathbb{F}^{v}\right) \otimes V_{\mathbb{W}}\right)\right) .
\end{gathered}
$$

For any $n$ there is a natural embedding $\mathfrak{s l}\left(V_{n}\right) \rightarrow \mathfrak{s p}\left(V_{n} \oplus V_{n}^{*}\right)$, and these embeddings define an embedding $\mathfrak{s l}_{\infty} \rightarrow \mathfrak{s p}_{\infty}$. We have

$$
\operatorname{Ann}_{\mathrm{U}\left(\mathfrak{s p}_{\infty}\right)}\left(\mathbf{S} \cdot\left(V_{\infty} \otimes \mathbb{F}^{v}\right) \otimes V_{\mathbb{W}}\right) \cap \mathrm{U}\left(\mathfrak{s l}_{\infty}\right)=I(2 v+1, \mathbb{F}),
$$

where $\mathbb{F}$ stands for the c.l.s. corresponding to the augmentation ideal, and therefore the image of $\operatorname{Var}\left(\operatorname{Ann}_{\mathrm{U}\left(\mathfrak{s p}_{\infty}\right)}\left(\mathbf{S} \cdot\left(V_{\infty} \otimes \mathbb{F}^{v}\right) \otimes V_{\mathbb{W}}\right)\right)$ under the projection $\mathfrak{s p}_{\infty}^{*} \rightarrow \mathfrak{s l}_{\infty}^{*}$ lies in $\mathfrak{s l}_{\infty}^{\leq 2 v+1}$. Hence, by Lemma 4.9 ,

$$
\operatorname{Var}\left(\operatorname{Ann}_{\mathrm{U}\left(\mathfrak{s p}_{\infty}\right)}\left(\mathbf{S} \cdot\left(V_{\infty} \otimes \mathbb{F}^{v}\right) \otimes V_{\mathbb{W}}\right)\right) \subset \mathfrak{s p}_{\infty}^{\leq 2 v+1} .
$$

This shows that $\operatorname{Var}\left(\operatorname{Ann}_{\mathrm{U}\left(\mathfrak{s p}_{\infty}\right)}\left(\mathbf{S} \cdot\left(V_{\infty} \otimes \mathbb{F}^{v}\right) \otimes V_{\mathbb{W}}\right)\right)=\mathfrak{s p}_{\infty}^{\leq 2 v+1}$.

\section{REFERENCES}

[BZh] A. A. Baranov, A. G. Zhilinskii, Diagonal direct limits of simple Lie algebras, Comm. Algebra 27 (1999), 2749-2766.

[B] A. A. Baranov, Finitary Simple Lie Algebras, Journal of Algebra 219(1999), 299-329.

[BS] A.A. Baranov, H. Strade, Finitary Lie algebras, Journal of Algebra 254 (2002) 173-211.

[Jo] A. Joseph, The minimal orbit in a simple Lie algebra and its associated maximal ideal, Ann. sc. de l'École Norm. Sup., Sér 4, 9:1(1976), 1-29. 
[AnPo] E. M. Andreev, V. L. Popov, The stationary subgroups of points in general position in a representation space of a semisimple Lie group (Russian), Funkcional. Anal. i Prilozen. 5: 4 (1971), 1-8.

[Bor] W. Borho, Über Schichten halbeinfacher Lie-Algebren, Invent. Math. 65 (1981/82), 283-317.

[Vi] Érnest Vinberg, Commutative homogeneous spaces and co-isotropic symplectic actions, Russian Math. Surv. 56(2001), 1-60.

[CM] David Collingwood, William McGovern, Nilpotent orbits in semisimple Lie algebras, Van Nostrand Reinhold Math. Ser., Van Nostrand Reinhold Co., New York, 1993.

[Zh1] A. G. Zhilinskii, Coherent systems of representations of inductive families of simple complex Lie algebras, (Russian) preprint of Academy of Belarussian SSR, ser. 38(438), Minsk, 1990.

[Zh2] A. G. Zhilinskii, Coherent finite-type systems of inductive families of non-diagonal inclusions, (Russian) Dokl. Acad. Nauk Belarusi 36:1(1992), 9-13, 92.

[Zh3] A. G. Zhilinskii, On the lattice of ideals in the universal enveloping algebra of a diagonal Lie algebra, preprint, Minsk, 2011.

[DP] I. Dimitrov, I. Penkov, Locally semisimple and maximal subalgebras of the finitary Lie algebras $\mathfrak{g l}(\infty), \mathfrak{s l}(\infty), \mathfrak{s o}(\infty)$, and $\mathfrak{s p}(\infty)$, Journal of Algebra 322(2009), 2069-2081.

[Dy] E. B. Dynkin, Semisimple subalgebras of semisimple Lie algebras (Russian), Mat. Sbornik N.S., 30(72) (1952), 349-462. English: AMS Translations 6(1957), 111-244.

[VP] É. B. Vinberg, V. L. Popov, Invariant theory (Russian) Algebraic geometry, 4 (Russian), Itogi Nauki i Tekhniki, Akad. Nauk SSSR, Vsesoyuz. Inst. Nauchn. i Tekhn. Inform., Moscow, $1989,137-315$.

Ivan Penkov (on leave from Jacobs University Bremen): Yale University, Department of Mathematics 10, Hillhouse Ave., New Haven, CT 06510, USA

E-mail address: i.penkov@jacobs-university.de

Alexey Petukhov: Institute for information transmission problems, Bolshoy KaretNIY 19-1, Moscow 127994, RussiA

E-mail address: alex--2@yandex.ru 